\title{
Three-sphere swimmer in a nonlinear viscoelastic medium
}

\author{
Mark P. Curtis* \\ Oxford Centre for Collaborative Applied Mathematics, Mathematical Institute, University of Oxford, Oxford OX1 3LB, United Kingdom
}

Eamonn A. Gaffney ${ }^{\dagger}$

Centre for Mathematical Biology, Mathematical Institute, University of Oxford, Oxford OXI 3LB, United Kingdom

(Received 26 October 2012; published 10 April 2013)

\begin{abstract}
A simple model for a swimmer consisting of three colinearly linked spheres attached by rods and oscillating out of phase to break reciprocal motion is analyzed. With a prescribed forcing of the rods acting on the three spheres, the swimming dynamics are determined analytically in both a Newtonian Stokes fluid and a zero Reynolds number, nonlinear, Oldroyd-B viscoelastic fluid with Deborah numbers of order one (or less), highlighting the effects of viscoelasticity on the net displacement of swimmer. For instance, the model predicts that the three-sphere swimmer with a sinusoidal, but nonreciprocal, forcing cycle within an Oldroyd-B representation of a polymeric Boger fluid moves a greater distance with enhanced efficiency in comparison with its motility in a Newtonian fluid of the same viscosity. Furthermore, the nonlinear contributions to the viscoelastic constitutive relation, while dynamically nontrivial, are predicted a posteriori to have no effect on swimmer motility at leading order, given a prescribed forcing between spheres.
\end{abstract}

DOI: 10.1103/PhysRevE.87.043006

PACS number(s): 47.63.mf, 47.50.Cd, 47.15.G-

\section{INTRODUCTION}

Modeling the motion and flow fields produced by shape changes and active filament bending in effectively zero Reynolds number media is of considerable importance in developing our understanding of complex biological processes, including the movement of bacteria during biofilm initiation, the beating of cilia for the induction of mucosal flows $[1,2]$, and flagellated cellular swimming, for instance sperm motility [3]. In particular, mammalian sperm must be able to penetrate media with viscoelastic rheology within the female reproductive tract, for example cervical mucus [4]. Assessing motility in mucus analogues is a common sperm diagnostic [5], illustrating the importance of low Reynolds number swimming dynamics in non-Newtonian media.

Here we analytically study the dynamics of a simplified swimmer, the Najafi-Golestanian three-sphere system [6], in a nonlinear rheological medium at effectively zero Reynolds number, which will allow us to highlight the effects of viscoelasticity on the relationships between swimmer strokes, applied forces, and motility. By enabling direct analytical investigations, and thus an understanding on the dynamics within the entire parameter space associated with the range of validity of the model, the simple swimmer models provide a testing ground for controlled experiments [7]. In addition, they can provide useful tests for the validation of numerical codes capable of considering more complex swimmers and rheologies. They can also contribute to understanding the impact of surfaces and cellular interactions in low Reynolds number swimming (e.g., [8]). Furthermore, simple swimmer models potentially offer the prospect of a simpler system to enhance our understanding of cellular swimming.

\footnotetext{
*curtis@maths.ox.ac.uk

${ }^{\dagger}$ gaffney@maths.ox.ac.uk; also at The Centre for Human Reproductive Science, Birmingham Women's NHS Foundation Trust, Edgbaston, Birmingham, United Kingdom.
}

Detailed studies have explored different swimming strokes and techniques in viscoelastic environments to investigate the effects of viscoelasticity on numerous swimmers. The first study known to the authors, by Fulford and Katz [9], considered filament swimmers with low amplitude sinusoidal beat patterns in a linearly viscoelastic fluid using a generalization of resistive-force theory, which is logarithmically accurate in expansions of the filament aspect ratio for Newtonian fluids [10]. The resulting predictions were faster swimming speeds in the linearly viscoelastic fluid given the same viscosity and energy expenditure [9]; improvements for swimming in rheological media have also been illustrated by swimming sheets with large undulations in nonlinear viscoelastic media [11]. In contrast, other studies accounting for a fixed forcing of a filament, modeled with resistive-force theory, suggest viscoelasticity can decrease the swimming speed for infinite filaments [12,13]. Also of immense interest are the observations that Purcell's scallop theorem [14] is broken in rheological media, both by simple, axisymmetric-squirming spheres at high Deborah number (the ratio of the viscoelastic relaxation time scale to the swimming stroke time scale) [15] and also small amplitude undulations of an infinite filament [13].

The effect of viscoelasticity has not, however, been investigated in relation to the three-sphere swimmer described in Sec. II A, although work has been implemented for the swimmer in a Newtonian fluid, [6,16-18] and a shear-thinning fluid, [19]. While Lauga [15] presents a technique of calculating the average displacement for a general swimmer in a viscoelastic environment, given the solid-body (undeforming) motion and associated force in a Newtonian flow field, these calculations contain lengthy expressions when considering the three-sphere swimmer, especially given the multiple length scales required to treat this swimmer analytically even in the Newtonian setting. Thus we proceed in this paper with an asymptotic approach generalizing the Newtonian calculations $[6,16,18]$, exploring how viscoelasticity alters swimming, especially when the forces driving the spheres, and hence driving the swimming motility, are prescribed. 


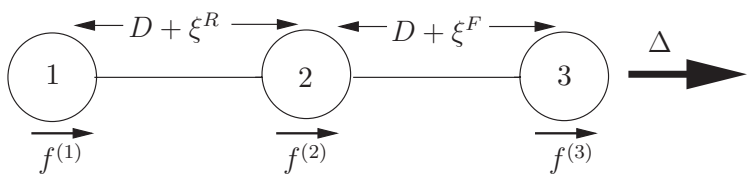

FIG. 1. The Najafi-Golestanian three-sphere swimmer. Three colinear, identical, neutrally buoyant spheres are connected by rods of mean length $D$ with periodic, but, in general, nonreciprocal, oscillations given by $\xi^{R}(t)$ and $\xi^{F}(t)$. The force acting on each sphere is denoted $\mathbf{f}^{(\alpha)}(t)$, where $\alpha \in\{1,2,3\}$, and the net displacement over one beat cycle is denoted $\Delta$ for the Newtonian problem and $\Delta_{v}$ for the viscoelastic analog.

\section{MODEL FORMULATION}

\section{A. Governing equations}

The three-sphere swimmer [6] consists of three colinear, identical, neutrally buoyant spheres, each of radius $a$, where the axis of motion of the swimmer is taken to be the $z$ axis with unit vector $\mathbf{e}_{z}$, connected by thin, dynamically negligible rods with oscillating lengths $D+\xi^{R}(t)$ between spheres 1 and 2 (the rear) and $D+\xi^{F}(t)$ between spheres 2 and 3 (the front); see Fig. 1.

The force acting on each sphere is denoted $\mathbf{f}^{(\alpha)}(t)$, where $\alpha \in\{1,2,3\}$; these are the equal and opposite forces arising, for example, due to motor devices located inside, and acting on, the spheres themselves. In order to compare the swimming progress and efficiency of the swimmer in a viscoelastic fluid to that in a Newtonian fluid, periodic forces on sphere 1 and 3 are prescribed, denoted $\mathbf{f}^{(1)}(t)$ and $\mathbf{f}^{(3)}(t)$ respectively. with beat frequency $\omega$ and a phase difference $\phi$ present between them to break time reversibility of the stroke. Note that $\mathbf{f}^{(2)}(t)$ is automatically determined upon assuming a zero Reynolds number regime as this requires zero net force; see Eq. (12).

The sphere separations vary about their mean length $D$ via the displacements $\xi^{R}(t)$ and $\xi^{F}(t)$, induced by the prescribed, periodic forces. Upon neglecting any initial transient behavior of the swimmer by assuming it has settled into periodic motility, the displacements are themselves periodic with beat frequency $\omega$. Sphere $\alpha$, where $\alpha \in\{1,2,3\}$, has center $\mathbf{x}^{(\alpha)}(t)$ and velocity $\mathbf{U}^{(\alpha)}(t)$. Thus,

$$
\begin{aligned}
\mathbf{U}^{(2)}-\mathbf{U}^{(1)} & =\dot{\xi}^{R} \mathbf{e}_{z}, \quad \mathbf{U}^{(3)}-\mathbf{U}^{(2)}=\dot{\xi}^{F} \mathbf{e}_{z}, \\
\left|\mathbf{x}^{(2)}-\mathbf{x}^{(1)}\right| & =D+\xi^{R}, \quad\left|\mathbf{x}^{(3)}-\mathbf{x}^{(2)}\right|=D+\xi^{F},
\end{aligned}
$$

where a superscript dot denotes differentiation with respect to time.

To investigate the effects of viscoelasticity on the net swimmer displacement per beat cycle, $\Delta$, we consider a viscoelastic fluid of density $\rho$, dynamic viscosity $\mu$ moving at velocity $\mathbf{u}(\mathbf{x}, t)$ with modified pressure (assuming a neutrally buoyant swimmer [20]) $p(\mathbf{x}, t)$, measured at a field point $\mathbf{x}$ and time $t$. Prior to assuming a low Reynolds number environment, the fluid dynamics are governed by the principles of the Navier-Stokes equations, though the deviatoric stress tensor, $\boldsymbol{\tau}(\mathbf{x}, t)$ that we focus on is that of an Oldroyd-B model [21], which, for example, can encompass a polymeric Boger fluid [22]. The governing equations are

$$
\nabla \cdot \mathbf{u}=0, \quad-\nabla p+\nabla \cdot \boldsymbol{\tau}=\rho\left(\frac{\partial \mathbf{u}}{\partial t}+(\mathbf{u} \cdot \nabla) \mathbf{u}\right),
$$

with

$$
\begin{aligned}
\boldsymbol{\tau} & +\lambda\left[\frac{\partial \boldsymbol{\tau}}{\partial t}+\mathbf{u} \cdot \nabla \boldsymbol{\tau}-\kappa_{1}\left([\nabla \mathbf{u}]^{T} \cdot \boldsymbol{\tau}+\boldsymbol{\tau} \cdot[\nabla \mathbf{u}]\right)\right] \\
& =\mu \mathcal{E}+\mu \gamma\left[\frac{\partial \mathcal{E}}{\partial t}+\mathbf{u} \cdot \nabla \mathcal{E}-\kappa_{2}\left([\nabla \mathbf{u}]^{T} \cdot \mathcal{E}+\mathcal{E} \cdot[\nabla \mathbf{u}]\right)\right]
\end{aligned}
$$

where $\lambda, \gamma$ are relaxation times and

$$
\mathcal{E}:=(\nabla \mathbf{u})^{T}+\nabla \mathbf{u},
$$

is the rate of strain tensor. For an Oldroyd-B fluid in particular, $\kappa_{1}=\kappa_{2}=1$ so that the tensor derivatives are upper convected; for a representation of a polymeric fluid, the constant $\gamma=\lambda \mu_{s} /\left(\mu_{s}+\mu_{p}\right)$ is taken to represent the retardation time, where $\mu_{s}$ and $\mu_{p}$ are the respective viscosities of the solvent and polymer [23,24]. This framework also includes the parameters $\gamma=0, \kappa_{1}=\kappa_{2}=1$, which is an upper convected Maxwell fluid, used to describe a polymer melt [22]. The results below readily, and immediately, generalize to other parameters in the constitutive relation, for example $\gamma=0$, $\kappa_{1}=\kappa_{2}=-1$, a lower convected Maxwell fluid [23,25].

Finally, note that the three-sphere swimmer kinematics are coupled to the fluid mechanics via no-slip boundary conditions, which are imposed on all sphere surfaces,

$$
\mathbf{u}=\mathbf{U}^{(\alpha)}(t) \quad \text { on } \quad\left|\mathbf{x}-\mathbf{x}^{(\alpha)}\right|=a, \quad \alpha \in\{1,2,3\} .
$$

\section{B. Nondimensionalization and simplifications}

The geometry of the model incorporates 3 length scales: the sphere radius, $a$, the mean length of the rod, $D$, and the amplitude of the rod displacements, $b$, so that $\left|\xi^{R}(t)\right| \leqslant b$ and $\left|\xi^{F}(t)\right| \leqslant b$. All length scales will be nondimensionalized by $a$, with time nondimensionalized by $1 / \omega$, where $\omega$ is the frequency of the periodic rod displacements, $\xi^{R}(t)$ and $\xi^{F}(t)$.

Noting a pragmatic constraint that the swimmer mechanism could comprise a motor device within the spheres themselves, one might readily envisage that $b<a$; below we further assume $\epsilon:=b / a \ll 1$ so that the amplitude of the swimmer motion is much smaller than the radius of the spheres. In addition, the average distance of separation between the spheres due to the rods, $D$, is assumed to be much greater than the radii of the spheres, $a$, so that $\delta:=a / D \ll 1$; in summary we have a separation of length scales

$$
b \ll a \ll D .
$$

One can see that, by nondimensionalizing lengths with $a, \xi^{R}(t)$ and $\xi^{F}(t)$ are $O(\epsilon)$ in dimensionless variables and thus

$$
\mathbf{x}=a \hat{\mathbf{x}}, \quad \nabla=\hat{\nabla} / a, \quad t=\hat{t} / \omega, \quad \xi^{\beta}(t)=\epsilon a \hat{\xi}^{\beta}(\hat{t}),
$$

where $\beta \in\{R, F\}$ and hatted variables are both dimensionless and $O(1)$. Note that, dimensionally, we will see that the velocity field outside a sphere for the parameter regime of interest is algebraic in $1 / r$, thus, near the sphere surface, the scaling $\nabla=\hat{\nabla} / a$ ensures $\hat{\nabla} \hat{\mathbf{u}} \sim O(1)$, which can be confirmed a posteriori. Equations (1) suggest that the scale of the sphere velocities induced by the rod oscillations, $U$, is given by $U=b \omega=\epsilon a \omega$ the velocity field generated in the surrounding fluid is driven by this oscillatory motion and thus 
inherits the same scale. Hence,

$$
\mathbf{U}^{(\alpha)}=\epsilon a \omega \hat{\mathbf{U}}^{(\alpha)}, \quad \frac{d \mathbf{x}^{(\alpha)}}{d \hat{t}}=\epsilon \hat{\mathbf{U}}^{(\alpha)}, \quad \mathbf{u}=\epsilon a \omega \hat{\mathbf{u}},
$$

where, again, hatted variables are dimensionless and $O(1)$. Thus, dropping the hat notation and working in dimensionless variables henceforth, the equations in (1) for the swimmer motion become

$$
\begin{aligned}
\mathbf{U}^{(2)}-\mathbf{U}^{(1)} & =\dot{\xi}^{R} \mathbf{e}_{z}, \quad \mathbf{U}^{(3)}-\mathbf{U}^{(2)}=\dot{\xi}^{F} \mathbf{e}_{z}, \\
\left|\mathbf{x}^{(2)}-\mathbf{x}^{(1)}\right| & =1 / \delta+\epsilon \xi^{R}, \quad\left|\mathbf{x}^{(3)}-\mathbf{x}^{(2)}\right|=1 / \delta+\epsilon \xi^{F} .
\end{aligned}
$$

The governing fluid equations (2) and (3) are rewritten in a frame centered on sphere $\alpha$, so that the origin is at $\mathbf{x}^{(\alpha)}(t)$. Variables in this noninertial frame are denoted by a superscript $\alpha$. Motivated by the observation that the deviatoric stress tensor in a Newtonian fluid is proportional to the gradient of the velocity, we have that $\tau$ and the pressure, $p$, are nondimensionalized by $\mu \epsilon \omega$ to ensure they are $O(1)$. Subsequently, forces are nondimensionalized by $\mu \epsilon \omega a^{2}$.

We assume all length scales are sufficiently small to neglect the advective Reynolds number, Re. By assigning an appropriate sphere radius and oscillation frequency, such that $\omega a^{2} \ll \mu / \rho$, the frequency Reynolds number, $\operatorname{Re}_{\omega}:=$ $\rho \omega a^{2} / \mu$, can also be taken to be negligible, thus allowing techniques associated with Stokes flow to be used. In the upcoming asymptotic analysis, an a posteriori check implies that one requires $\operatorname{Re}, \operatorname{Re}_{\omega} \sim O\left(\delta^{3}\right)$ or less. Also, we consider a regime in which the Deborah numbers $\Lambda:=\omega \lambda$ and $\Gamma:=\omega \gamma$ [26] are $O(1)$, implying that the relaxation time and retardation time of the fluid are comparable to the time scale of the swimmer oscillations. In particular, the order-one Deborah number regime has already been observed numerically to give rise to a peak in swimming efficiency of free swimming sheets [11] and thus is of clear interest in understanding swimmer-rheological interactions.

By nondimensionalizing, the governing equations become

$$
\begin{aligned}
\nabla \cdot \mathbf{u}^{(\alpha)}= & 0, \quad-\nabla p^{(\alpha)}+\nabla \cdot \boldsymbol{\tau}^{(\alpha)}=\mathbf{0}, \\
\left(1+\Lambda \frac{\partial}{\partial t}\right) \boldsymbol{\tau}^{(\alpha)}= & \left(1+\Gamma \frac{\partial}{\partial t}\right) \mathcal{E}^{(\alpha)} \\
& -\epsilon\left[\mathbf{u}^{(\alpha)} \cdot \nabla\left[\Lambda \boldsymbol{\tau}^{(\alpha)}-\Gamma \mathcal{E}^{(\alpha)}\right]\right. \\
& -\left\{\left(\nabla \mathbf{u}^{(\alpha)}\right)^{T} \cdot\left[\Lambda \boldsymbol{\tau}^{(\alpha)}-\Gamma \mathcal{E}^{(\alpha)}\right]\right. \\
& \left.+\left[\Lambda \boldsymbol{\tau}^{(\alpha)}-\Gamma \mathcal{E}^{(\alpha)}\right] \cdot \nabla \mathbf{u}^{(\alpha)}\right\} \\
& \left.-\mathbf{U}^{(\alpha)} \cdot \nabla\left[\Lambda \boldsymbol{\tau}^{(\alpha)}-\Gamma \mathcal{E}^{(\alpha)}\right]\right], \\
\mathcal{E}^{(\alpha)}:= & \left(\nabla \mathbf{u}^{(\alpha)}\right)^{T}+\nabla \mathbf{u}^{(\alpha)} .
\end{aligned}
$$

The no slip boundary conditions on the spheres are given by

$$
\mathbf{u}^{(\alpha)}=\mathbf{U}^{(\beta)}-\mathbf{U}^{(\alpha)} \text { on }\left|\mathbf{x}-\mathbf{x}^{(\beta)}\right|=1, \quad \beta \in\{1,2,3\},
$$

where $\mathbf{x}^{(\beta)}=\mathbf{0}$ when $\beta=\alpha$. An important consequence of the inertialess regime is that the sum of all the forces on the spheres is zero,

$$
\sum_{\alpha=1}^{3} \mathbf{f}^{(\alpha)}=\mathbf{0}
$$

which will be exploited below.

\section{NEWTONIAN FLUID CASE}

\section{A. Approximating the Newtonian flow field}

Before considering the full viscoelastic model given by equations (9)-(11), an initial insight can be gained by considering the Newtonian problem $[6,16,18]$, whereby the three-sphere swimmer is immersed in a viscous Newtonian fluid with no viscoelastic properties. We reproduce expressions for the average displacement after one beat cycle, in agreement with existing results [18,27], but we consistently account for higher orders of the sphere interactions to obtain the force on the spheres in a Newtonian fluid, noting that deducing a net motion over a beat cycle necessitates the consideration of higher-order corrections. These results will also be required when examining the viscoelastic case.

Using the same notation as before in a coordinate frame centered in sphere $\alpha$, the Newtonian governing equations are

$$
\begin{aligned}
-\nabla p^{(\alpha)}+\nabla \cdot \boldsymbol{\tau}^{(\alpha)} & =\mathbf{0}, \quad \nabla \cdot \mathbf{u}^{(\alpha)}=0, \\
\boldsymbol{\tau}^{(\alpha)} & =\mathcal{E}^{(\alpha)}:=\left(\nabla \mathbf{u}^{(\alpha)}\right)^{T}+\nabla \mathbf{u}^{(\alpha)}, \\
\mathbf{u}^{(\alpha)} & =\mathbf{U}^{(\beta)}-\mathbf{U}^{(\alpha)} \text { on }\left|\mathbf{x}-\mathbf{x}^{(\beta)}\right|=1,
\end{aligned}
$$

with

$$
\begin{aligned}
\mathbf{U}^{(2)}-\mathbf{U}^{(1)} & =\dot{\xi}^{R} \mathbf{e}_{z}, \quad \mathbf{U}^{(3)}-\mathbf{U}^{(2)}=\dot{\xi}^{F} \mathbf{e}_{z}, \\
\frac{1}{\left|\mathbf{x}^{(2)}-\mathbf{x}^{(1)}\right|} & =\delta\left(1+\delta \epsilon \xi^{R}\right)^{-1}, \\
\frac{1}{\left|\mathbf{x}^{(3)}-\mathbf{x}^{(2)}\right|} & =\delta\left(1+\delta \epsilon \xi^{F}\right)^{-1},
\end{aligned}
$$

and boundary conditions, where $\beta \in\{1,2,3\}$,

$$
\mathbf{u}^{(\alpha)}=\mathbf{U}^{(\beta)}-\mathbf{U}^{(\alpha)} \text { on }\left|\mathbf{x}-\mathbf{x}^{(\beta)}\right|=1 .
$$

The fluid environment is inertialess, given the Reynolds number, Re, has been assumed to be negligible. This ensures there are no inertial terms in the governing equations (13), allowing variables to be treated as quasi-steady in the Newtonian case. With the subsequent viscoelastic case, there is the potential for initial, transient behavior, though we only consider solutions where this transient behavior arising from initial conditions in time have decayed and the swimmer has settled into periodic beating. Hence variables in the boundary conditions, such as $\mathbf{U}^{(\alpha)}(t)$, can be considered with time, $t$, simply behaving as a parameter.

The Newtonian Stokes flow field due to a single translating sphere of unit radius, sphere velocity $\mathbf{U}$ and center $\mathbf{y}$ in an infinite domain can be represented via a linear combination of a Stokeslet, $\mathbf{S}(\mathbf{x}, \mathbf{y})$, and potential dipole, $\mathbf{D}(\mathbf{x}, \mathbf{y})$ such that

$$
\mathbf{u}(\mathbf{x})=\mathbf{G}(\mathbf{x}, \mathbf{y}) \mathbf{U}
$$

where

$$
\mathbf{G}(\mathbf{x}, \mathbf{y}):=\frac{3}{4} \mathbf{S}(\mathbf{x}, \mathbf{y})-\frac{1}{4} \mathbf{D}(\mathbf{x}, \mathbf{y})
$$

and

$$
\mathbf{G}(\mathbf{x}, \mathbf{y})=\mathbf{I} \quad \text { on } \quad|\mathbf{x}-\mathbf{y}|=1
$$


This motivates an initial postulation, $\mathbf{u}_{A}^{(\alpha)}(\mathbf{x})$, for the velocity flow field centered in sphere $\alpha$ as

$$
\mathbf{u}_{A}^{(\alpha)}(\mathbf{x})=-\mathbf{U}^{(\alpha)}+\sum_{k=1}^{3} \mathbf{G}\left(\mathbf{x}, \mathbf{x}^{(k)}\right) \mathbf{U}^{(k)} .
$$

However, (19) does not exactly satisfy the boundary conditions (15). Nonetheless, if we take $\mathbf{x}$ on the surface of sphere $\beta$ so that $\mathbf{x}=\mathbf{x}^{(\beta)}+\mathbf{e}_{r}^{(\beta)}$, where $\mathbf{e}_{r}$ is a unit radial vector from the center of sphere $\beta$ to its surface, we see that $\left|\mathbf{x}^{(\gamma)}-\mathbf{x}^{(\beta)}\right| \sim O(1 / \delta)$ for $\gamma \neq \beta$. Thus one can expand the singularities evaluated on the sphere surfaces as

$$
\mathbf{G}\left(\mathbf{x}^{(\beta)}+\mathbf{e}_{r}^{(\beta)}, \mathbf{x}^{(\gamma)}\right)=\mathbf{G}^{(\beta, \gamma)}+\left(\mathbf{e}_{r}^{(\beta)} \cdot \nabla\right) \mathbf{G}^{(\beta, \gamma)}+\cdots,
$$

where $\mathbf{G}^{(\beta, \gamma)}:=\mathbf{G}\left(\mathbf{x}^{(\beta)}, \mathbf{x}^{(\gamma)}\right)$, for example,

$$
\begin{aligned}
\mathbf{G}^{(2,1)} & =\frac{3}{4} \mathbf{S}\left(\mathbf{x}^{(2)}, \mathbf{x}^{(1)}\right)-\frac{1}{4} \mathbf{D}\left(\mathbf{x}^{(2)}, \mathbf{x}^{(1)}\right), \\
& =\frac{3}{4}\left(\frac{\mathbf{I}+\mathbf{e}_{z} \otimes \mathbf{e}_{z}}{\left|\mathbf{x}^{(2)}-\mathbf{x}^{(1)}\right|}\right)-\frac{1}{4}\left(\frac{-\mathbf{I}+\mathbf{e}_{z} \otimes \mathbf{e}_{z}}{\left|\mathbf{x}^{(2)}-\mathbf{x}^{(1)}\right|^{3}}\right), \\
& \sim \frac{3 \delta}{4}\left(\mathbf{I}+\mathbf{e}_{z} \otimes \mathbf{e}_{z}\right)\left(1-\delta \epsilon \xi^{R}\right)+O\left(\delta^{3}\right) .
\end{aligned}
$$

To proceed, the $O\left(\delta^{3}\right)$ terms (and hence the potential dipole) will be neglected as this level of accuracy is not required below. In order to retain the $O\left(\delta^{2} \epsilon\right)$ terms in Eq. (21) while neglecting $O\left(\delta^{3}\right)$ terms, we impose that $\epsilon \gg \delta$ : physically this requires that the ratio of a sphere radius compared to the separation of the spheres is much less than the ratio of the amplitude of rod oscillations to the sphere radius. We could expand variables in $\epsilon$ as well, but, given that we are neglecting $O\left(\delta^{3}\right)$ and the next $\epsilon$ term is $O\left(\delta^{3} \epsilon\right)$, there are no further $\epsilon$ contributions other than those in Eq. (21). Also note that the next term in the Stokeslet expansion in Eq. (21) is order $O\left(\delta^{3} \epsilon^{2}\right)$ and hence negligible.

Expansion (21) highlights how terms of the form $\mathbf{G}^{(\beta, \gamma)}$ include constant $O(\delta)$ terms and quasisteady time dependent, though treated as constant, $O\left(\delta^{2} \epsilon\right)$ terms; these are denoted $\delta \boldsymbol{K}^{(\beta, \gamma)}$ and $\delta^{2} \epsilon \boldsymbol{H}^{(\beta, \gamma)}$ respectively. Terms of the form $\left(\mathbf{e}_{r}^{(\beta)}\right.$. $\nabla) \mathbf{G}^{(\beta, \gamma)}$ in Eq. (20) will only have $O\left(\delta^{2}\right)$ contributions, neglecting $O\left(\delta^{3}\right)$, denoted $\delta^{2} \boldsymbol{\Gamma}^{\left(\beta, \gamma ; \mathbf{e}_{r}^{\beta}\right)}$, where the superscript $\mathbf{e}_{r}^{\beta}$ refers to the linear spatial dependence on $\mathbf{e}_{r}^{(\beta)}$. Any higherorder derivatives in Eq. (20) are $O\left(\delta^{3}\right)$ and hence negligible. Formally,

$$
\begin{gathered}
\boldsymbol{K}^{(\beta, \gamma)}:=\lim _{\delta \rightarrow 0} \frac{\mathbf{G}^{(\beta, \gamma)}}{\delta}, \\
\boldsymbol{H}^{(\beta, \gamma)}:=\lim _{\delta, \epsilon \rightarrow 0}\left(\frac{\mathbf{G}^{(\beta, \gamma)}-\boldsymbol{K}^{(\beta, \gamma)}}{\delta^{2} \epsilon}\right), \\
\boldsymbol{\Gamma}^{\left(\beta, \gamma ; \mathbf{e}_{r}^{\beta}\right)}:=\lim _{\delta \rightarrow 0} \frac{\left(\mathbf{e}_{r}^{(\beta)} \cdot \nabla\right) \mathbf{G}^{(\beta, \gamma)}}{\delta^{2}} .
\end{gathered}
$$

Our initial postulation for the flow field, $\mathbf{u}_{A}^{(\alpha)}(\mathbf{x})$, evaluated on the boundary of sphere $\beta$, is thus

$$
\begin{aligned}
\mathbf{u}_{A}^{(\alpha)}\left(\mathbf{x}^{(\beta)}+\mathbf{e}_{r}^{(\beta)}\right) \sim & \mathbf{U}^{(\beta)}-\mathbf{U}^{(\alpha)}+\sum_{\gamma \neq \beta}\left(\delta \mathbf{K}^{(\beta, \gamma)}+\delta^{2} \epsilon \boldsymbol{H}^{(\beta, \gamma)}\right. \\
& \left.+\delta^{2} \boldsymbol{\Gamma}^{\left(\beta, \gamma ; \mathbf{e}_{r}^{\beta}\right)}\right) \mathbf{U}^{(\gamma)}+O\left(\delta^{3}\right)
\end{aligned}
$$

where $\beta \neq \alpha$ and $\gamma$ ranges from 1 to 3 .

\section{B. Higher-order corrections in $\delta$}

The approximate flow field $\mathbf{u}_{A}^{(\alpha)}(\mathbf{x})$ is based upon an initial postulation in which spheres are separated by very long rods and does not satisfy the exact boundary conditions in Eq. (15); instead, the boundary conditions contain additional $O(\delta)$ terms, as illustrated by equation (25). In order to correct $\mathbf{u}_{A}^{(\alpha)}(\mathbf{x})$ to within $O\left(\delta^{3}\right)$ (and hence negligible) errors, we deal with both the constant and spatially varying terms in Eq. (25) separately by introducing correction variables $\mathbf{u}^{(\alpha)}=\mathbf{u}_{A}^{(\alpha)}+\delta \mathbf{u}_{B}^{(\alpha)}+\delta^{2} \hat{\mathbf{u}}^{(\alpha)}$ and $p=p_{A}+\delta p_{B}+\delta^{2} \hat{p}$ which both satisfy Stokes equations, but differ in their boundary conditions. These variables can still contain higher-order $\delta$ and $\epsilon$ terms; explicitly $\mathbf{u}_{B}^{(\alpha)}$ is constant on the surface of sphere $\beta$, and given by

$$
\mathbf{u}_{B}^{(\alpha)}\left(\mathbf{x}^{(\beta)}+\mathbf{e}_{r}^{(\beta)}\right)=-\sum_{\gamma \neq \beta}\left(\mathbf{K}^{(\beta, \gamma)}+\delta \epsilon \boldsymbol{H}^{(\beta, \gamma)}\right) \mathbf{U}^{(\gamma)},
$$

where $\beta \in\{1,2,3\}$, while $\hat{\mathbf{u}}^{(\alpha)}$ is spatially varying on the surface of sphere $\beta$ and given by

$$
\hat{\mathbf{u}}^{(\alpha)}\left(\mathbf{x}^{(\beta)}+\mathbf{e}_{r}^{(\beta)}\right)=-\sum_{\gamma \neq \beta} \boldsymbol{\Gamma}^{\left(\beta, \gamma / \mathbf{e}_{r}^{\beta}\right)} \mathbf{U}^{(\gamma)} .
$$

As the equation determining $\mathbf{u}_{B}^{(\alpha)}(\mathbf{x})$ has constant boundary conditions, we can similarly construct an approximate solution based on infinite-domain singularities, which again will not satisfy the boundary conditions exactly, requiring further corrections at higher orders; thus we are effectively using the technique of reflections, as discussed in Ref. [28]. Piecing the solution back together gives

$$
\begin{aligned}
\mathbf{u}^{(\alpha)}(\mathbf{x})= & -\mathbf{U}^{(\alpha)}+\sum_{\gamma=1}^{3} \mathbf{G}\left(\mathbf{x}, \mathbf{x}^{(\gamma)}\right)\left[\mathbf{U}^{(\gamma)}-\sum_{\nu \neq \gamma}\left\{\left(\delta \mathbf{K}^{(\gamma, v)}\right.\right.\right. \\
& \left.\left.\left.+\delta^{2} \epsilon \boldsymbol{H}^{(\gamma, \nu)}\right) \mathbf{U}^{(v)}+\delta^{2} \mathbf{K}^{(\gamma, v)}\left(\sum_{\chi \neq \nu} \mathbf{K}^{(\nu, \chi)} \mathbf{U}^{(\chi)}\right)\right\}\right] \\
& +\delta^{2} \hat{\mathbf{u}}^{(\alpha)}+O\left(\delta^{3}\right) .
\end{aligned}
$$

It will be shown that calculating $\hat{\mathbf{u}}^{(\alpha)}$ explicitly will not be required, though its evaluation can be reduced to the problem of a sphere in an inertialess shear flow, which possesses a well-known solution [28,29].

Note that in the very far field, where the centers of spheres 1 and 3 may be considered to be asymptotically close to the center of sphere 2, the leading-order behavior of the swimmer is that of Stokeslets. Given it is a force-free swimmer, these in fact reduce to two Stokeslet doublets, representing an asymmetric swimmer. However, if one were to average the flow field over a period in time, the periodic, singularity weightings, $\mathbf{U}^{(\gamma)}$, ensure the Stokeslet-doublet weightings have a net value of zero over a period. This implies higher orders are needed to describe the time-averaged swimmer, which is therefore a symmetric, quadrupole swimmer. 


\section{Newtonian force calculations}

The force acting on sphere $\alpha$ due to the viscous drag and the interactions of the other spheres is calculated by integrating the component of the stress tensor in the $\mathbf{e}_{r}^{(\alpha)}$ direction over the sphere surface, $|\mathbf{x}|=1$. Pozrikidis [29] derives a Faxen relation for flows past a sphere with sufficiently rapid decay conditions, all of which are satisfied by the Stokeslets and potential dipoles used in Eq. (17), stating the force acting on the sphere is given by

$$
\mathbf{f}^{(\alpha)}=\frac{3}{2} \int_{|\mathbf{X}|=1} \mathbf{u}^{(\alpha) \text { background }} d S,
$$

where $\mathbf{u}^{(\alpha) \text { background }}$ is the flow if one were to neglect the disturbance flow due to the presence of sphere $\alpha$. This consists of the background flow, $-\boldsymbol{U}^{(\alpha)}$, arising from fixing the coordinate frame in sphere $\alpha$ and the flow due to the interactions of the other spheres with themselves. The surface integral in Eq. (29) highlights that this background flow need only be evaluated on the surface of sphere $\alpha$, and any $\boldsymbol{\Gamma}$ terms integrate to zero around the surface of sphere $\alpha$ via a symmetry argument. Thus, using (29), neglecting $O\left(\delta^{3}\right)$,

$$
\begin{aligned}
\mathbf{f}^{(\alpha)}= & -6 \pi \mathbf{U}^{(\alpha)}+6 \pi \sum_{\gamma \neq \alpha}\left[\delta \mathbf{K}^{(\alpha, \gamma)}\left(\mathbf{U}^{(\gamma)}-\delta \mathbf{K}^{(\gamma, \sigma)} \mathbf{U}^{(\sigma)}\right)\right. \\
& \left.+\delta^{2} \epsilon \mathbf{H}^{(\alpha, \gamma)} \mathbf{U}^{(\gamma)}\right],
\end{aligned}
$$

where, for each $\gamma, \sigma$ labels the remaining sphere such that $\sigma \neq \gamma, \alpha$. Equation (30) relates the force on sphere $\alpha$ to the sphere velocities and can be used to determine the net displacement over one beat cycle. The analog of equation (30) for a viscoelastic fluid will be derived in Sec. IV C.

\section{Average swimmer displacement in Newtonian fluid}

Deriving the Newtonian force on each sphere in terms of the velocities of the spheres (30), allows for an average displacement of the whole swimmer over one beat cycle to be calculated. As a reference point for the displacement, the center of sphere 2, the middle sphere of the swimmer, is chosen. By inserting (30) into the Stokes flow force balance (12), along with

$$
\mathbf{U}^{(1)}=\mathbf{U}^{(2)}-\dot{\xi}^{R} \mathbf{e}_{z}, \quad \mathbf{U}^{(3)}=\mathbf{U}^{(2)}+\dot{\xi}^{F} \mathbf{e}_{z},
$$

an equation for $\mathbf{U}^{(2)}$ is obtained. As $\mathbf{U}^{(2)}=U^{(2)} \mathbf{e}_{z}$, this equation is scalar and of the form,

$$
Q U^{(2)}=B,
$$

where, after some algebra,

$$
\begin{aligned}
Q=3\left(1-\frac{5 \delta}{2}+3 \delta^{2}+\frac{5 \delta^{2} \epsilon}{4}\left(\xi^{R}+\xi^{F}\right)\right), \\
B=\left(1-\frac{9 \delta}{4}+\frac{27 \delta^{2}}{8}\right)\left(\dot{\xi}^{R}-\dot{\xi}^{F}\right) \\
+\frac{3 \delta^{2} \epsilon}{8}\left(\xi^{F} \dot{\xi}^{R}-\xi^{R} \dot{\xi}^{F}+5\left[\xi^{R} \dot{\xi}^{R}-\xi^{F} \dot{\xi}^{F}\right]\right) .
\end{aligned}
$$

Motivated by Eq. (34), perturbation expansions of the following form are used,

$$
\begin{aligned}
U^{(2)} & =U_{0,0}^{(2)}+\delta U_{1,0}^{(2)}+\delta^{2} U_{2,0}^{(2)}+\delta^{2} \epsilon U_{2,1}^{(2)}+O\left(\delta^{3}\right), \\
\xi^{R, F} & =\xi_{0,0}^{R, F}+\delta \xi_{1,0}^{R, F}+\delta^{2} \xi_{2,0}^{R, F}+\delta^{2} \epsilon \xi_{2,1}^{R, F}+O\left(\delta^{3}\right),
\end{aligned}
$$

which, upon solving at each order, gives

$$
\begin{aligned}
U^{(2)} \sim & \frac{1}{3}\left(\dot{\xi}^{R}-\dot{\xi}^{F}\right)\left\{1+\frac{\delta}{4}+\delta^{2}\right\}+\delta^{2} \epsilon\left[\frac{5}{24}\left(\xi^{R} \dot{\xi}^{R}-\xi^{F} \dot{\xi}^{F}\right)\right. \\
& \left.+\frac{7}{24}\left(\xi^{R} \dot{\xi}^{F}-\xi^{F} \dot{\xi}^{R}\right)\right]+O\left(\delta^{3}\right) .
\end{aligned}
$$

This is consistent with existing work [18,27] if one were to expand in $a / D$ in their notation and set $\epsilon=1$ in the above; note that Earl et al. [27] present corrected displacement expressions to the original work by Najafi and Golestanian [6]. The net displacement of the swimmer over one beat cycle, $\Delta$, is given by

$$
\Delta=\int_{0}^{2 \pi} U^{(2)} d t
$$

where $2 \pi$ is the period of the beat, recalling that time has been nondimensionalized with beat frequency. For periodic rod displacements $\xi^{R}(t)$ and $\xi^{F}(t)$, we note that all the terms up to, and including, $O\left(\delta^{2}\right)$ in Eq. (36) integrate to zero in Eq. (37) when calculating $\Delta$, thus justifying the need to retain $O\left(\delta^{2} \epsilon\right)$, leaving

$$
\Delta=\frac{7 \delta^{2} \epsilon}{24} \int_{0}^{2 \pi}\left(\xi^{R} \dot{\xi}^{F}-\xi^{F} \dot{\xi}^{R}\right) d t .
$$

Redimensionalizing Eq. (38) gives, assuming dimensional variables,

$$
\Delta=\frac{7 a}{24 D^{2}} \int_{0}^{2 \pi / \omega}\left(\xi^{R} \dot{\xi}^{F}-\xi^{F} \dot{\xi}^{R}\right) d t .
$$

For an intuitive understanding of Eq. (38), in a reference frame centered in sphere $\alpha$, the surface force acting on sphere $\alpha, \mathbf{f}^{(\alpha)}$, must be due to any contributions of a background flow on the surface of sphere $\alpha$. This background flow includes the term $-\mathbf{U}^{(\alpha)}(t)$ due to the reference frame, along with all the flows produced by the interactions of the other spheres with themselves, i.e., spheres $\gamma \in\{1,2,3\}$ where $\gamma \neq \alpha$. Approximating the other spheres as a combination of a Stokeslet and potential dipole, $\mathbf{G}(\mathbf{x}, \mathbf{y})$, the total background flow on the surface of sphere $\alpha$ is hence

$$
\begin{aligned}
& \mathbf{u}^{(\alpha) \text { background }}\left(\mathbf{x}^{(\alpha)}(t)+\mathbf{e}_{r}^{(\alpha)}\right) \\
& \approx-\mathbf{U}^{(\alpha)}(t)+\sum_{\gamma \neq \alpha} \mathbf{G}\left(\mathbf{x}^{(\alpha)}(t)+\mathbf{e}_{r}^{(\alpha)}, \mathbf{x}^{(\gamma)}(t)\right) \mathbf{U}^{(\gamma)}(t), \\
& \approx-\mathbf{U}^{(\alpha)}(t)+\sum_{\gamma \neq \alpha} \mathbf{S}\left(\mathbf{x}^{(\alpha)}(t), \mathbf{x}^{(\gamma)}(t)\right) \mathbf{U}^{(\gamma)}(t),
\end{aligned}
$$

where the Stokeslet, $\mathbf{S}(\mathbf{x}, \mathbf{y})$, approximates $\mathbf{G}(\mathbf{x}, \mathbf{y})$, given the potential-dipolar contributions are of higher order. Using 
Eq. (9),

$$
\begin{aligned}
\mathbf{S}\left(\mathbf{x}^{(\alpha)}(t), \mathbf{x}^{(\gamma)}(t)\right) & \sim O\left(\frac{1}{\left|\mathbf{x}^{(\gamma)}-\mathbf{x}^{(\alpha)}\right|}\right), \\
& \sim \delta A_{1}+\delta^{2} A_{2}+\delta^{2} \epsilon B\left[\xi^{R}(t), \xi^{F}(t)\right],
\end{aligned}
$$

for constants $A_{1}$ and $A_{2}$ and a function, $B\left[\xi^{R}, \xi^{F}\right]$, linear in $\xi^{R}(t)$ and $\xi^{F}(t)$. A posteriori motivated by Eq. (41), the neglected dipoles in Eq. (40) were $O\left(\delta^{3}\right)$, while any terms that were not linear in $\mathbf{e}_{r}^{(\alpha)}$ from the Taylor series are also $O\left(\delta^{3}\right)$. Thus, integrating (40) around sphere $\alpha$, as is necessary to find $\mathbf{f}^{(\alpha)}$, just adds a constant so that $\mathbf{f}^{(\alpha)} \propto \mathbf{u}^{(\alpha) \text { background }}\left(\mathbf{x}^{(\alpha)}(t)\right)$ to $O\left(\delta^{3}\right)$ corrections. Knowing the sum of all forces is zero and that $\mathbf{U}^{(\gamma)}(t)$ relates to $\mathbf{U}^{(\alpha)}(t)$ via $\dot{\xi}^{R}(t)$ and $\dot{\xi}^{F}(t)$ from the swimmer geometry, an expression for $\mathbf{U}^{(\alpha)}(t)$ is obtainable from Eq. (40). Moreover, this expression for $\mathbf{U}^{(\alpha)}(t)$ can be written asymptotically in the same form as the expanded Stokeslet from Eq. (41), albeit with terms multiplied by linear combinations of $\dot{\xi}^{R}(t)$ and $\dot{\xi}^{F}(t)$. Time averaging over one beat cycle gives nonzero contributions at $O\left(\epsilon \delta^{2}\right)$, as these terms depend nonlinearly on $B\left(\xi^{R}, \xi^{F}\right)$ multiplied by $\xi(t)$ and $\dot{\xi}(t)$.

Further, the resulting quadratic combination takes the form:

$$
\begin{aligned}
& B_{1} \dot{\xi}^{R}(t) \xi^{R}(t)+B_{2} \dot{\xi}^{F}(t) \xi^{F}(t)+B_{3} \dot{\xi}^{F}(t) \xi^{R}(t) \\
& \quad+B_{4} \dot{\xi}^{R}(t) \xi^{F}(t) .
\end{aligned}
$$

Clearly the first two terms integrate to zero when time averaged over a period, and, by the antisymmetry of the $\operatorname{map}\left(\xi^{R}, \xi^{F}\right) \rightarrow$ $\left(\xi^{F}, \xi^{R}\right)$ implying $\Delta \rightarrow-\Delta$, we must have that $B_{4}=-B_{3}$. This is in agreement with Eq. (38), and gives its form to within a constant factor.

The dimensionless, net displacement (38) is currently written in terms of the rod displacements, $\xi^{R}(t)$ and $\xi^{F}(t)$. In Sec. II A, however, the model considers a swimmer with prescribed forces (the motility problem), not prescribed rod displacements, to highlight the influence of viscoelasticity on swimming progress and efficiency for a swimmer with a predetermined actuation force [12]. Thus, to write Eq. (38) in terms of the forces on sphere 1 and $3, \mathbf{f}^{(1)}=f^{(1)} \mathbf{e}_{z}$ and $\mathbf{f}^{(3)}=f^{(3)} \mathbf{e}_{z}$, where $\mathbf{f}^{(2)}$ is deduced from Eq. (12), we substitute (36) and (31) into Eq. (30) to obtain

$$
\left(\begin{array}{l}
f^{(1)} \\
f^{(3)}
\end{array}\right)=\pi \mathbf{M}\left(\begin{array}{l}
\dot{\xi}^{R} \\
\dot{\xi}^{F}
\end{array}\right)
$$

where

$$
\begin{aligned}
\mathbf{M}= & \left(\begin{array}{cc}
4 & 2 \\
-2 & -4
\end{array}\right)+\delta\left(\begin{array}{cc}
4 & \frac{1}{2} \\
-\frac{1}{2} & -4
\end{array}\right)+\delta^{2}\left(\begin{array}{cc}
-\frac{61}{8} & -\frac{47}{8} \\
\frac{47}{8} & \frac{61}{8}
\end{array}\right) \\
& +\delta^{2} \epsilon\left(\begin{array}{cc}
\xi^{F}-5 \xi^{R} & -\frac{\xi^{F}+\xi^{R}}{4} \\
\frac{\xi^{F}+\xi^{R}}{4} & 5 \xi^{F}-\xi^{R}
\end{array}\right)+O\left(\delta^{3}\right) .
\end{aligned}
$$

The inverse is calculated perturbatively, giving

$$
\left(\begin{array}{l}
\dot{\xi}^{R} \\
\dot{\xi}^{F}
\end{array}\right)=\frac{1}{\pi} \mathbf{N}\left(\begin{array}{l}
f^{(1)} \\
f^{(3)}
\end{array}\right)
$$

where

$$
\begin{aligned}
\mathbf{N}= & \left(\begin{array}{cc}
\frac{1}{3} & \frac{1}{6} \\
-\frac{1}{6} & -\frac{1}{3}
\end{array}\right)+\delta\left(\begin{array}{cc}
-\frac{4}{9} & -\frac{1}{72} \\
\frac{1}{72} & \frac{4}{9}
\end{array}\right)+\delta^{2}\left(\begin{array}{cc}
\frac{311}{216} & \frac{71}{432} \\
-\frac{71}{432} & -\frac{311}{216}
\end{array}\right) \\
& +\delta^{2} \epsilon\left(\begin{array}{ll}
\frac{5 \xi^{R}-\xi^{F}}{9} & \frac{\xi^{F}+\xi^{R}}{144} \\
-\frac{\left(\xi^{F}+\xi^{R}\right)}{144} & \frac{\xi^{R}-5 \xi^{F}}{9}
\end{array}\right)+O\left(\delta^{3}\right) .
\end{aligned}
$$

Note that the inverse is a constant matrix up to, and including, $O\left(\delta^{2}\right)$; the $\xi^{F}(t)$ and $\xi^{R}(t)$ terms do not appear in the inverse matrix until $O\left(\delta^{2} \epsilon\right)$. Upon using (45) to rewrite $\mathbf{U}^{(2)}$ in Eq. (36) in terms of forces, then integrating over one beat period assuming nondimensional $2 \pi$-periodic forcing,

$$
\Delta=\frac{29 \delta^{2} \epsilon}{432 \pi^{2}} \int_{0}^{2 \pi}\left(g^{(3)} \dot{g}^{(1)}-g^{(1)} \dot{g}^{(3)}\right) d t,
$$

where $\dot{g}^{(\alpha)}:=f^{(\alpha)}$ and $g^{(\alpha)}=\int^{t} \dot{g}^{(\alpha)}(\bar{t}) d \bar{t}$, allowing the net displacement over one beat cycle (47) to be written solely in terms of the forces on the spheres. Thus, by prescribing $2 \pi$ periodic internal forces of the three-sphere swimmer instead of rod displacements, an expression for the distance traveled by the swimmer in one beat cycle is obtained. Also, by Green's theorem, $\Delta$ is proportional to the area enclosed in the $\left(g^{(1)}, g^{(3)}\right)$ plane during a period by $g^{(1)}$ and $g^{(3)}$.

Redimensionalizing Eq. (47) gives, assuming dimensional variables,

$$
\Delta=\frac{29}{432 \pi^{2} \mu^{2} D^{2} a} \int_{0}^{2 \pi / \omega}\left(g^{(3)} \dot{g}^{(1)}-g^{(1)} \dot{g}^{(3)}\right) d t,
$$

where time scales are implicit in the force integrals.

As an example, prescribing dimensionless, internal forces on spheres 1 and 3 as

$$
\mathbf{f}^{(1)}=\sin (t) \mathbf{e}_{z}, \quad \mathbf{f}^{(3)}=\sin (t-\phi) \mathbf{e}_{z},
$$

where the phase difference $\phi$ is included to break time reversibility, implies a dimensionless displacement over one beat cycle of

$$
\Delta=-\frac{29 \delta^{2} \epsilon \sin (\phi)}{216 \pi}
$$

\section{VISCOELASTIC FLUID CASE}

\section{A. Viscoelastic asymptotic analysis}

We now extend existing work $[18,27]$ by investigating the full viscoelastic model from Sec. II A, nondimensionalized with respect to a fixed frame in sphere $\alpha$, so that the equations of motion are given by

$$
\begin{aligned}
\nabla \cdot \mathbf{u}^{(\alpha)}= & 0, \quad-\nabla p^{(\alpha)}+\nabla \cdot \boldsymbol{\tau}^{(\alpha)}=\mathbf{0} \\
\left(1+\Lambda \frac{\partial}{\partial t}\right) \boldsymbol{\tau}^{(\alpha)}= & \left(1+\Gamma \frac{\partial}{\partial t}\right) \mathcal{E}^{(\alpha)} \\
& -\epsilon\left[\mathbf{u}^{(\alpha)} \cdot \nabla\left[\Lambda \boldsymbol{\tau}^{(\alpha)}-\Gamma \mathcal{E}^{(\alpha)}\right]\right. \\
& -\left\{\left(\nabla \mathbf{u}^{(\alpha)}\right)^{T} \cdot\left[\Lambda \boldsymbol{\tau}^{(\alpha)}-\Gamma \mathcal{E}^{(\alpha)}\right]\right. \\
& \left.+\left[\Lambda \boldsymbol{\tau}^{(\alpha)}-\Gamma \mathcal{E}^{(\alpha)}\right] \cdot \nabla \mathbf{u}^{(\alpha)}\right\} \\
& \left.-\mathbf{U}^{(\alpha)} \cdot \nabla\left[\Lambda \boldsymbol{\tau}^{(\alpha)}-\Gamma \mathcal{E}^{(\alpha)}\right]\right] \\
\mathcal{E}^{(\alpha)}:= & \left(\nabla \mathbf{u}^{(\alpha)}\right)^{T}+\nabla \mathbf{u}^{(\alpha)}
\end{aligned}
$$


with

$$
\begin{aligned}
\mathbf{U}^{(2)}-\mathbf{U}^{(1)} & =\dot{\xi}^{R} \mathbf{e}_{z}, \quad \mathbf{U}^{(3)}-\mathbf{U}^{(2)}=\dot{\xi}^{F} \mathbf{e}_{z}, \\
\frac{1}{\left|\mathbf{x}^{(2)}-\mathbf{x}^{(1)}\right|} & =\delta\left(1+\delta \epsilon \xi^{R}\right)^{-1}, \\
\frac{1}{\left|\mathbf{x}^{(3)}-\mathbf{x}^{(2)}\right|} & =\delta\left(1+\delta \epsilon \xi^{F}\right)^{-1},
\end{aligned}
$$

and boundary conditions

$$
\mathbf{u}^{(\alpha)}=\mathbf{U}^{(\beta)}-\mathbf{U}^{(\alpha)} \text { on }\left|\mathbf{x}-\mathbf{x}^{(\beta)}\right|=1, \quad \beta \in\{1,2,3\},
$$

where $\mathbf{x}^{(\beta)}=\mathbf{0}$ when $\beta=\alpha$. The difference between these equations compared with those of the Newtonian problem occur in the deviatoric stress tensor definition, in particular via the addition of an $O(1)$ partial time derivative, recalling the Deborah numbers $\Lambda, \Gamma$ are assumed to be $O(1)$ while nonlinear terms are $O(\epsilon)$.

All dimensionless variables are expanded in powers of $\epsilon$, such that a variable $\mathbf{q}^{(\alpha)}$, say, has the expansion

$$
\mathbf{q}^{(\alpha)} \sim \mathbf{q}_{0}^{(\alpha)}+\epsilon \mathbf{q}_{1}^{(\alpha)}+O\left(\epsilon^{2}\right),
$$

where $\mathbf{q}_{0}^{(\alpha)}$ and $\mathbf{q}_{1}^{(\alpha)}$ will depend on $\delta$, although only up to $O\left(\delta^{2}\right)$ given that $O\left(\delta^{3}\right)$ terms are negligible; see the discussion following Eq. (21). The leading-order problem in $\epsilon$ is given by

$$
\begin{aligned}
\nabla \cdot \mathbf{u}_{0}^{(\alpha)} & =0, \quad-\nabla p_{0}^{(\alpha)}+\nabla \cdot \boldsymbol{\tau}_{0}^{(\alpha)}=\mathbf{0}, \\
\mathcal{L}\left[\boldsymbol{\tau}_{0}^{(\alpha)}\right] & =\mathcal{E}_{0}^{(\alpha)}, \quad \mathcal{E}_{0}^{(\alpha)}=\left(\nabla \mathbf{u}_{0}^{(\alpha)}\right)^{T}+\nabla \mathbf{u}_{0}^{(\alpha)}, \\
\mathbf{U}_{0}^{(2)}-\mathbf{U}_{0}^{(1)} & =\dot{\xi}_{0}^{R} \mathbf{e}_{z}, \quad \mathbf{U}_{0}^{(3)}-\mathbf{U}_{0}^{(2)}=\dot{\xi}_{0}^{F} \mathbf{e}_{z}, \\
\left(\frac{1}{\left|\mathbf{x}^{(2)}-\mathbf{x}^{(1)}\right|}\right)_{0} & =\delta, \quad\left(\frac{1}{\left|\mathbf{x}^{(3)}-\mathbf{x}^{(2)}\right|}\right)_{0}=\delta, \\
\mathbf{u}_{0}^{(\alpha)} & =\mathbf{U}_{0}^{(\beta)}-\mathbf{U}_{0}^{(\alpha)} \text { on }\left|\mathbf{x}-\mathbf{x}^{(\beta)}\right|=1, \\
\beta & \in\{1,2,3\},
\end{aligned}
$$

where the temporal operator $\mathcal{L}$ is defined as

$$
\mathcal{L}:=\left(1+\Gamma \frac{\partial}{\partial t}\right)^{-1}\left(1+\Lambda \frac{\partial}{\partial t}\right) .
$$

Care is required to uniquely define the inverse in Eq. (56) as it is contingent on the initial conditions. We consider only solutions where transient behavior arising from initial conditions in time have decayed and the swimmer has settled into periodic beating to ensure uniqueness, thus the solutions are perfectly periodic. Other than the temporal operator, $\mathcal{L}$, acting on the deviatoric stress tensor, the viscoelastic equations at leading (zero) order are essentially those of the Newtonian fluid, and the variable transformation

$$
\mathbf{w}_{0}^{(\alpha)}=\mathbf{u}_{0}^{(\alpha)}, \quad q_{0}^{(\alpha)}=\mathcal{L}\left[p_{0}^{(\alpha)}\right], \quad \boldsymbol{\tau}_{w_{0}}^{(\alpha)}=\mathcal{L}\left[\boldsymbol{\tau}_{0}^{(\alpha)}\right],
$$

where

$$
\boldsymbol{\tau}_{w_{0}}^{(\alpha)}:=\left(\nabla \mathbf{w}_{0}^{(\alpha)}\right)^{T}+\nabla \mathbf{w}_{0}^{(\alpha)},
$$

casts the above problem exactly into the Newtonian case. Hence $\mathbf{w}_{0}^{(\alpha)}$ is given by Newtonian flow field solution calculated earlier (28), but with $\epsilon=0$ and velocity $\mathbf{U}^{(\alpha)}$ taken at leading order, so that

$$
\begin{aligned}
\mathbf{w}_{0}^{(\alpha)}(\mathbf{x})= & -\mathbf{U}_{0}^{(\alpha)}+\sum_{\gamma=1}^{3} \mathbf{G}\left(\mathbf{x}, \mathbf{x}^{(\gamma)}\right)\left[\mathbf{U}_{0}^{(\gamma)}-\sum_{\nu \neq \gamma}\left\{\delta \mathbf{K}^{(\gamma, \nu)} \mathbf{U}_{0}^{(\nu)}\right\}\right] \\
& +\left.\delta^{2} \hat{\mathbf{u}}^{(\alpha)}\right|_{\epsilon=0}+O\left(\delta^{3}\right) .
\end{aligned}
$$

Note that a surface integral around sphere $\alpha$ is over a timeindependent domain (given the frame is fixed about the center of sphere $\alpha$ ) and thus commutes with the temporal operator $\mathcal{L}$. Hence the viscoelastic force acting on sphere $\alpha$ at leading order, denoted $\mathbf{f}_{0}^{(\alpha)}(t)$, and the Newtonian force on sphere $\alpha$ at leading order in $\epsilon$, denoted $\mathbf{f}_{w_{0}}^{(\alpha)}(t)$, satisfy the relation

$$
\mathcal{L}\left[\mathbf{f}_{0}^{(\alpha)}(t)\right]=\mathbf{f}_{w_{0}}^{(\alpha)}(t),
$$

where $\mathbf{f}_{w_{0}}^{(\alpha)}(t)$ is given by the Newtonian force calculated earlier (30), again with the added change of setting $\epsilon=0$ and velocity $\mathbf{U}^{(\alpha)}$ taken at leading order, so that

$$
\mathbf{f}_{w_{0}}^{(\alpha)}(t)=-6 \pi \mathbf{U}_{0}^{(\alpha)}+6 \pi \sum_{k \neq \alpha}\left[\delta \mathbf{K}^{(\alpha, k)}\left(\mathbf{U}_{0}^{(k)}-\delta \mathbf{K}^{(k, \sigma)} \mathbf{U}_{0}^{(\sigma)}\right)\right]
$$

This gives a relationship for the viscoelastic force at leading order in terms of the Newtonian force from Eq. (30); we now proceed to correct this relationship at $O(\epsilon)$.

\section{B. Higher-order analysis in $\epsilon$}

The $O(\epsilon)$ equations are given by

$$
\begin{aligned}
\nabla \cdot \mathbf{u}_{1}^{(\alpha)} & =0, \quad-\nabla p_{1}^{(\alpha)}+\nabla \cdot \boldsymbol{\tau}_{1}^{(\alpha)}=\mathbf{0}, \\
\mathcal{L}\left[\boldsymbol{\tau}_{1}^{(\alpha)}\right]+\boldsymbol{\eta}^{(\alpha)} & =\mathcal{E}_{1}^{(\alpha)}, \quad \mathcal{E}_{1}^{(\alpha)}=\left(\nabla \mathbf{u}_{1}^{(\alpha)}\right)^{T}+\nabla \mathbf{u}_{1}^{(\alpha)}, \\
\mathbf{U}_{1}^{(2)}-\mathbf{U}_{1}^{(1)} & =\dot{\xi}_{1}^{R} \mathbf{e}_{z}, \quad \mathbf{U}_{1}^{(3)}-\mathbf{U}_{1}^{(2)}=\dot{\xi}_{1}^{F} \mathbf{e}_{z}, \\
\left(\frac{1}{\left|\mathbf{x}^{(2)}-\mathbf{x}^{(1)}\right|}\right)_{1} & =\delta^{2} \xi_{0}^{R}, \quad\left(\frac{1}{\left|\mathbf{x}^{(3)}-\mathbf{x}^{(2)}\right|}\right)_{1}=-\delta^{2} \xi_{0}^{F}, \\
\mathbf{u}_{1}^{(\alpha)} & =\mathbf{U}_{1}^{(\beta)}-\mathbf{U}_{1}^{(\alpha)} \text { on }\left|\mathbf{x}-\mathbf{x}^{(\beta)}\right|=1, \quad \beta=1,2,3,
\end{aligned}
$$

where

$$
\begin{aligned}
\boldsymbol{\eta}^{(\alpha)}:= & \left(1+\Gamma \frac{\partial}{\partial t}\right)^{-1}\left[\mathbf{u}_{0}^{(\alpha)} \cdot \nabla\left[\Lambda \boldsymbol{\tau}_{0}^{(\alpha)}-\Gamma \mathcal{E}_{0}^{(\alpha)}\right]\right. \\
& -\left\{\left(\nabla \mathbf{u}_{0}^{(\alpha)}\right)^{T} \cdot\left[\Lambda \boldsymbol{\tau}_{0}^{(\alpha)}-\Gamma \mathcal{E}_{0}^{(\alpha)}\right]\right. \\
& \left.+\left[\Lambda \boldsymbol{\tau}_{0}^{(\alpha)}-\Gamma \mathcal{E}_{0}^{(\alpha)}\right] \cdot \nabla \mathbf{u}_{0}^{(\alpha)}\right\} \\
& \left.-\mathbf{U}_{0}^{(\alpha)} \cdot \nabla\left[\Lambda \boldsymbol{\tau}_{0}^{(\alpha)}-\Gamma \mathcal{E}_{0}^{(\alpha)}\right]\right]
\end{aligned}
$$

Here, $\boldsymbol{\eta}^{(\alpha)}$ is a known function as $\boldsymbol{\tau}_{0}^{(\alpha)}$ and $\mathcal{E}_{0}^{(\alpha)}$ are defined in terms of the leading-order flow field $\mathbf{u}_{0}^{(\alpha)}$ from equations (55), where $\mathbf{u}_{0}^{(\alpha)}$ itself is given by Eq. (59), noting that $\mathbf{u}_{0}^{(\alpha)}=\mathbf{w}_{0}^{(\alpha)}$ from Eq. (57).

We split up the problem into a viscoelastic part, with velocity $\mathbf{v}_{1}^{(\alpha)}$, and a Newtonian part, with velocity $\mathbf{w}_{1}^{(\alpha)}$

$$
\begin{aligned}
\mathbf{u}_{1}^{(\alpha)} & =\mathbf{v}_{1}^{(\alpha)}+\mathbf{w}_{1}^{(\alpha)}, \quad \mathcal{L}\left[p_{1}^{(\alpha)}\right]=s_{1}^{(\alpha)}+q_{1}^{(\alpha)}, \\
\mathcal{L}\left[\boldsymbol{\tau}_{1}^{(\alpha)}\right] & =\left(\boldsymbol{\tau}_{v_{1}}^{(\alpha)}-\boldsymbol{\eta}^{(\alpha)}\right)+\boldsymbol{\tau}_{w_{1}}^{(\alpha)},
\end{aligned}
$$


where

$$
\boldsymbol{\tau}_{v_{1}}^{(\alpha)}:=\left(\nabla \mathbf{v}_{1}^{(\alpha)}\right)^{T}+\nabla \mathbf{v}_{1}^{(\alpha)}, \quad \boldsymbol{\tau}_{w_{1}}^{(\alpha)}:=\left(\nabla \mathbf{w}_{1}^{(\alpha)}\right)^{T}+\nabla \mathbf{w}_{1}^{(\alpha)},
$$

such that

$$
\begin{aligned}
& \nabla \cdot \mathbf{v}_{1}^{(\alpha)}=0, \quad-\nabla s_{1}^{(\alpha)}+\nabla \cdot \tau_{v_{1}}^{(\alpha)}=\nabla \cdot \eta^{(\alpha)}, \\
& \mathbf{v}_{1}^{(\alpha)}=\mathbf{0} \quad \text { on } \quad|\mathbf{x}|=1
\end{aligned}
$$

and

$$
\begin{aligned}
\nabla \cdot \mathbf{w}_{1}^{(\alpha)} & =0, \quad-\nabla q_{1}^{(\alpha)}+\nabla \cdot \boldsymbol{\tau}_{w_{1}}^{(\alpha)}=\mathbf{0}, \\
\mathbf{U}_{1}^{(2)}-\mathbf{U}_{1}^{(1)} & =\dot{\xi}_{1}^{R} \mathbf{e}_{z}, \quad \mathbf{U}_{1}^{(3)}-\mathbf{U}_{1}^{(2)}=\dot{\xi}_{1}^{F} \mathbf{e}_{z}, \\
\left(\frac{1}{\left|\mathbf{x}^{(2)}-\mathbf{x}^{(1)}\right|}\right)_{1} & =\delta^{2} \xi_{0}^{R}, \quad\left(\frac{1}{\left|\mathbf{x}^{(3)}-\mathbf{x}^{(2)}\right|}\right)_{1}=-\delta^{2} \xi_{0}^{F}, \\
\mathbf{w}_{1}^{(\alpha)} & =\left(\mathbf{U}_{1}^{(\beta)}-\mathbf{v}_{1}^{(\alpha)}\right)-\mathbf{U}_{1}^{(\alpha)} \quad \text { on } \quad\left|\mathbf{x}-\mathbf{x}^{(\beta)}\right|=1,
\end{aligned}
$$

with $\beta \in\{1,2,3\}$. In the Appendix we solve the $\mathbf{v}_{1}^{(\alpha)}$ equations (66) to show that

$$
\begin{array}{lll}
\mathbf{v}_{1}^{(\alpha)}=\mathbf{0} \quad \text { on } & |\mathbf{x}|=1, \quad \beta=\alpha, \\
\mathbf{v}_{1}^{(\alpha)} \sim \delta^{2} \mathbf{A}^{(\alpha)} & \text { on } \quad\left|\mathbf{x}-\mathbf{x}^{(\beta)}\right|=1, \quad \beta \neq \alpha .
\end{array}
$$

where $\mathbf{A}^{(\alpha)}$ is an $O(1)$ constant vector. The boundary condition for the $\mathbf{w}_{1}^{(\alpha)}$ problem (67) is thus

$$
\mathbf{w}_{1}^{(\alpha)}=\left(\mathbf{U}_{1}^{(\beta)}-\delta^{2} \mathbf{A}^{(\beta)}\right)-\mathbf{U}_{1}^{(\alpha)} \quad \text { on } \quad\left|\mathbf{x}-\mathbf{x}^{(\beta)}\right|=1,
$$

where $\beta \in\{1,2,3\}$; note this is constant on each sphere. Hence the $\mathbf{w}_{1}^{(\alpha)}$ problem above can be viewed as the $O(\epsilon)$ Newtonian problem (13) if one were to expand the Newtonian problem in $\epsilon$ and inspect the $O(\epsilon)$ equations, albeit with $\mathbf{U}_{1}^{(\beta)}$ replaced by $\mathbf{U}_{1}^{(\beta)}-\delta^{2} \mathbf{A}^{(\beta)}$ in the boundary conditions. It follows that the force in the $\mathbf{w}_{1}^{(\alpha)}$ problem (69), denoted $\mathbf{f}_{w_{1}}^{(\alpha)}(t)$, is the $O(\epsilon)$ contribution to the Newtonian force in Eq. (30) with the adjusted boundary conditions, so that

$$
\begin{aligned}
\mathbf{f}_{w_{1}}^{(\alpha)}(t)= & -6 \pi \mathbf{U}_{1}^{(\alpha)}+6 \pi \sum_{\gamma \neq \alpha}\left[\delta \mathbf{K}^{(\alpha, \gamma)}\left(\mathbf{U}_{1}^{(\gamma)}-\delta \mathbf{K}^{(\gamma, \sigma)} \mathbf{U}_{1}^{(\sigma)}\right)\right. \\
& \left.+\delta^{2} \mathbf{H}_{0}^{(\alpha, \gamma)} \mathbf{U}_{0}^{(\gamma)}\right],
\end{aligned}
$$

where $\mathbf{H}_{0}^{(\alpha, \gamma)}:=\mathbf{H}^{(\alpha, \gamma)}\left(\xi_{0}^{R}, \xi_{0}^{F}\right)$ from Eq. (23). Importantly, the extra contributions, $\delta^{2} \mathbf{A}^{(\beta)}$, do not contribute to the force in the $\mathbf{w}_{1}^{(\alpha)}$ problem as they are multiplied by $\delta$ in Eq. (70) to become $O\left(\delta^{3}\right)$ and hence negligible. Finally, we can show that the total force at $O(\epsilon)$ from both the $\mathbf{v}_{1}^{(\alpha)}$ and $\mathbf{w}_{1}^{(\alpha)}$ problems, denoted $\mathbf{f}_{1}^{(\alpha)}(t)$, is solely due to the force in the $\mathbf{w}_{1}^{(\alpha)}$ problem (69) above, $\mathbf{f}_{w_{1}}^{(\alpha)}(t)$, since the force in the $\mathbf{v}_{1}^{(\alpha)}$ problem (66), denoted $\mathbf{f}_{v_{1}}^{(\alpha)}(t)$, cancels with the surface integral of $\boldsymbol{\eta}^{(\alpha)}$ after a careful application of the divergence theorem and noting that $\eta^{(\alpha)}$ decays at infinity. Thus,

$$
\mathcal{L}\left[\mathbf{f}_{1}^{(\alpha)}(t)\right]=\mathbf{f}_{w_{1}}^{(\alpha)}(t)
$$

A dimensional analysis and similar procedure to the $O(\epsilon)$ problem for higher orders in $\epsilon$ allows one to deduce that the total force, up to $O\left(\epsilon^{k}\right)$ where $k \geqslant 2$, relates to the Newtonian force at that order, $\mathbf{f}_{w_{k}}^{(\alpha)}(t)$, by

$$
\mathcal{L}\left[\mathbf{f}^{(\alpha)}(t)\right]=\sum_{k} \epsilon^{k} \mathcal{L}\left[\mathbf{f}_{w_{k}}^{(\alpha)}(t)\right]+O\left(\delta^{3}\right) .
$$

Equations (61) and (70) ensure that

$$
\begin{aligned}
\mathcal{L}\left[\mathbf{f}^{(\alpha)}(t)\right]= & -6 \pi \mathbf{U}^{(\alpha)} \\
& +6 \pi \sum_{m \neq \alpha}\left[\delta \mathbf{K}^{(\alpha, m)}\left(\mathbf{U}^{(m)}-\delta \mathbf{K}^{(m, \sigma)} \mathbf{U}^{(m)}\right)\right. \\
& \left.+\epsilon \delta^{2} \mathbf{H}^{(\alpha, m)} \mathbf{U}^{(m)}\right]+O\left(\delta^{3}\right) .
\end{aligned}
$$

This relationship is similar to that of the Newtonian problem (30), but now includes the temporal operator $\mathcal{L}$ on the left-hand side. Equation (73) holds so long as $O\left(\delta^{3}\right)$ is negligible and $\delta \ll \epsilon$; see Sec. III A.

\section{Average swimmer displacement in a viscoelastic fluid}

The Stokes force balance gives, upon applying the temporal operator $\mathcal{L}$,

$$
\sum_{\gamma=1}^{3} \mathcal{L}\left[\mathbf{f}^{(\gamma)}\right]=\mathbf{0},
$$

which can be written in terms of sphere velocities using (73). One can write $\mathbf{U}^{(1)}$ and $\mathbf{U}^{(3)}$ in terms of $\mathbf{U}^{(2)}$ via (31), giving the same expression for $U^{(2)}$ in terms of rod displacements $\xi^{R}$ and $\xi^{F}$ as in Eq. (36); this is unsurprising given the direct equivalence of velocities in Eq. (57). Thus, the equation for the net displacement of the swimmer in one beat cycle in a viscoelastic fluid, $\Delta_{v}$, when written in terms of rod displacements is equal to the net displacement in a Newtonian fluid given by Eq. (38),

$$
\Delta_{v}=\frac{7 \delta^{2} \epsilon}{24} \int_{0}^{2 \pi}\left(\xi^{R} \dot{\xi}^{F}-\xi^{F} \dot{\xi}^{R}\right) d t,
$$

and in dimensional variables by Eq. (39). This highlights how, in viscoelastic fluid, prescribing any forms of displacement such as $\xi^{R}$ and $\xi^{F}$ will leave the results for net swimmer displacement unchanged, at least up to and including $O\left(\delta^{2} \epsilon\right)$ for the three-sphere swimmer.

However, rewriting the net displacement in terms of viscoelastic forces using Eq. (73) so that one can prescribe the internal forcing of the swimmer instead of displacements, effectively adds a temporal $\mathcal{L}$ operator to $\mathbf{f}^{(\alpha)}$, so that

$$
\left(\begin{array}{l}
\dot{\xi}^{R} \\
\dot{\xi}^{F}
\end{array}\right)=\frac{1}{\pi} \mathbf{N}\left(\begin{array}{c}
\mathcal{L}\left[f^{(1)}\right] \\
\mathcal{L}\left[f^{(3)}\right]
\end{array}\right)
$$

where $\mathbf{N}$ remains unchanged from Eq. (46), giving

$$
\Delta_{v}=\frac{29 \delta^{2} \epsilon}{432 \pi^{2}} \int_{0}^{2 \pi}\left(g_{\mathcal{L}}^{(3)} \dot{g}_{\mathcal{L}}^{(1)}-g_{\mathcal{L}}^{(1)} \dot{g}_{\mathcal{L}}^{(3)}\right) d t,
$$

where

$$
\begin{aligned}
\dot{g}_{\mathcal{L}}^{(\alpha)}(t) & :=\mathcal{L}\left[f^{(\alpha)}(t)\right], \\
& =\left(1+\Gamma \frac{\partial}{\partial t}\right)^{-1}\left(1+\Lambda \frac{\partial}{\partial t}\right) f^{(\alpha)}(t),
\end{aligned}
$$

and

$$
g_{\mathcal{L}}^{(\alpha)}(t)=\int^{t} \dot{g}_{\mathcal{L}}^{(\alpha)}(\bar{t}) d \bar{t}
$$


This illustrates that viscoelasticity does indeed have an effect on the fixed-actuation-force swimmer. For rods with internal forcings that are in phase, $f^{(1)}(t)=f^{(3)}(t)$, or antiphase, $f^{(1)}(t)=-f^{(3)}(t)$, the displacement from Eq. (77) is zero, as expected due to swimmer symmetry. One can note that, by Green's theorem, $\Delta_{v}$ is proportional to the area enclosed in the $\left(g_{\mathcal{L}}^{(1)}, g_{\mathcal{L}}^{(3)}\right)$ plane during a period by $g_{\mathcal{L}}^{(1)}$ and $g_{\mathcal{L}}^{(3)}$.

Redimensionalizing equation (77) gives, assuming dimensional variables,

$$
\Delta=\frac{29}{432 \pi^{2} \mu^{2} D^{2} a} \int_{0}^{2 \pi / \omega}\left(g_{\mathcal{L}}^{(3)} \dot{g}_{\mathcal{L}}^{(1)}-g_{\mathcal{L}}^{(1)} \dot{g}_{\mathcal{L}}^{(3)}\right) d t,
$$

where time scales are implicit in the force integrals.

We take the example again of prescribing dimensionless internal forces on spheres 1 and 3 by

$$
\mathbf{f}^{(1)}=\sin (t) \mathbf{e}_{z}, \quad \mathbf{f}^{(3)}=\sin (t-\phi) \mathbf{e}_{z},
$$

where the phase difference $\phi$ is included to break time reversibility. With the additional information that initial transient behavior has decayed so that the solution is perfectly periodic, allowing a unique inverse to be defined for the temporal operator, Eq. (77) gives

$$
\Delta_{v}=-\frac{29 \delta^{2} \epsilon}{216 \pi}\left(\frac{1+\Lambda^{2}}{1+\Gamma^{2}}\right) \sin (\phi) .
$$

From the definitions of the dimensionless constants, $\Lambda:=$ $\omega \lambda$ for relaxation time $\lambda$ and $\Gamma:=\omega \gamma$ for retardation time $\gamma$, where, for a polymeric fluid as discussed in Sec. II A, the constant $\gamma=\lambda \mu_{s} /\left(\mu_{s}+\mu_{p}\right)$, hence we see that $\Lambda>\Gamma$ so that the net displacement of a swimmer in polymeric viscoelastic fluid from Eq. (82) is greater than that in a Newtonian fluid [18]. These results also hold if either, or both, of the Deborah numbers $\Lambda$ and $\Gamma$ are much less than one. Explicit plots of the displacement for an upper convected Maxwell fluid, where $\Gamma=0$, are illustrated in Fig. 2 .

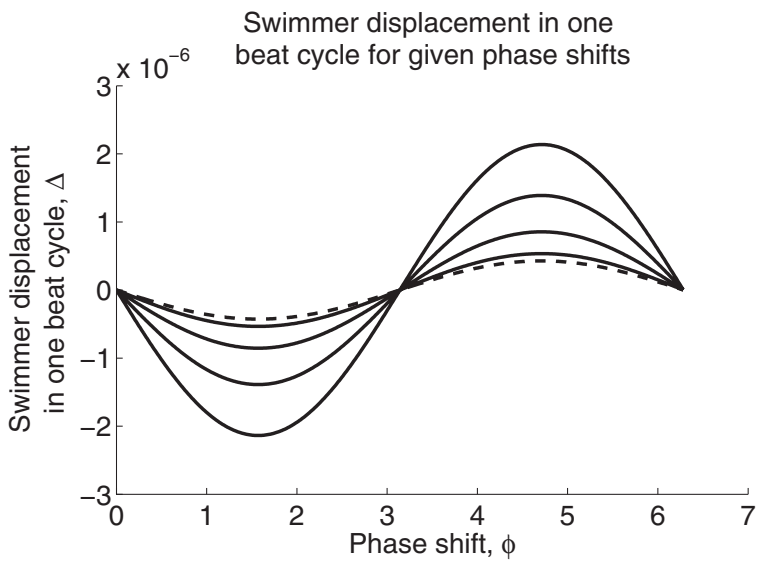

FIG. 2. The dimensionless net displacement over one beat cycle, $\Delta$ for a Newtonian fluid and $\Delta_{v}$ for the viscoelastic medium, is plotted as a function of rod phase difference, $\phi$, where the internal forcing of sphere 1 is $\sin (t)$ and of sphere 3 is $\sin (t-\phi)$. Here, $\epsilon=0.1$, $\delta=0.01, \Gamma=0$, as for an upper convected Maxwell fluid, and $\Lambda$ ranges from 0 , i.e., the Newtonian case (given by the dashed line) to 2 in steps of 0.5 , where each step increases the amplitude of the curve.

\section{Efficiency}

It is common, with simple swimmer models, to investigate the efficiency. Using Lighthill's definition of hydrodynamic efficiency, $E$, in terms of average power, $\bar{P}$, and average velocity, $\overline{\mathbf{U}}$ [30],

$$
E=6 \pi \mu \overline{\mathbf{U}}^{2} / \bar{P}
$$

where

$$
\begin{aligned}
\overline{\mathbf{U}} & :=\frac{1}{2 \pi} \int_{0}^{2 \pi} \mathbf{U}^{(2)}(t) d t=\frac{\Delta_{v} \mathbf{e}_{z}}{2 \pi}, \\
\bar{P} & :=\frac{1}{2 \pi} \int_{0}^{2 \pi} P(t) d t, \\
P(t) & :=\sum_{k=1}^{3} \mathbf{U}^{(k)}(t) \cdot \mathbf{f}^{(k)}(t), \\
& =-\mathbf{f}^{(1)}(t) \cdot \dot{\xi}^{R}(t) \mathbf{e}_{z}+\mathbf{f}^{(3)}(t) \cdot \dot{\xi}^{F}(t) \mathbf{e}_{z} .
\end{aligned}
$$

To simplify the power expression, we have used that

$$
\begin{aligned}
\mathbf{U}^{(1)} & =\mathbf{U}^{(2)}-\dot{\xi}^{R} \mathbf{e}_{z}, \quad \mathbf{U}^{(3)}=\mathbf{U}^{(2)}+\dot{\xi}^{F} \mathbf{e}_{z}, \\
\mathbf{0} & =\mathbf{f}^{(1)}+\mathbf{f}^{(2)}+\mathbf{f}^{(3)} .
\end{aligned}
$$

From Eq. (76), we can obtain $\dot{\xi}^{R}$ and $\dot{\xi}^{F}$ in terms of $\mathcal{L}\left[f^{(1)}\right]$ and $\mathcal{L}\left[f^{(3)}\right]$ and insert these into the expression for $P(t)$ in Eq. (84).

For the example

$$
\mathbf{f}^{(1)}=\sin (t) \mathbf{e}_{z}, \quad \mathbf{f}^{(3)}=\sin (t-\phi) \mathbf{e}_{z},
$$

we obtain

$$
\begin{aligned}
\bar{P}= & \frac{1}{432 \pi}\left(\frac{1+\Lambda \Gamma}{1+\Gamma^{2}}\right)\left[-2\left(72-96 \delta+311 \delta^{2}\right)\right. \\
& \left.+\left(-72+6 \delta-71 \delta^{2}\right) \cos \phi\right],
\end{aligned}
$$

which has a greater magnitude (given $\Lambda>\Gamma$ ) than the average Newtonian power (when $\Gamma=\Lambda=0$ ). To compare the hydrodynamic efficiency, (83), between the Newtonian and viscoelastic cases, $\overline{\mathbf{U}}$ can be written in terms of the net displacement per beat cycle, $\Delta_{v}$. The efficiency of the sinusoidal three-sphere swimmer with prescribed force actuation thus scales with

$$
\left(\frac{1+\Lambda^{2}}{1+\Gamma^{2}}\right)^{2} \frac{1+\Gamma^{2}}{1+\Lambda \Gamma}>1
$$

so that

$$
E_{\text {viscoelastic }}>E_{\text {Newtonian, }}
$$

demonstrating that this swimmer is more efficient when immersed in a viscoelastic fluid.

\section{CONCLUSION}

The distance displaced in one beat cycle of a three-linkedsphere swimmer has been investigated in both a Newtonian and a viscoelastic fluid environment, the latter with a deviatoric stress tensor described by a polymeric Oldroyd-B model, including an upper convected Maxwell model. The results also immediately generalize for other constitutive relation parameters in Eq. (3), such as those associated with a lower convected Maxwell fluid. 
The parameter regime is such that there are two small parameters, $\delta, \epsilon$. The former is the ratio of the sphere sizes to their separation, so that sphere interactions are via their far fields, enabling extensive simplification. The latter is the ratio of the sphere oscillation amplitudes to their radii. Its small size is arguably not unreasonable for self-contained forcing mechanisms within the spheres and entails the viscoelastic problem reduces to a forced Stokes problem at each order of an asymptotic expansion, sometimes referred to as the retarded motion expansion [26]. In addition, recall that $\delta \ll \epsilon$ is imposed in Sec. III A, which also is satisfied for a sufficiently large sphere separation. Finally, note that the requirement of effectively inertialess dynamics enforces sufficiently small length scales and also that $\rho \omega a^{2} / \mu \sim O\left(\delta^{3}\right)$ or less, where $\mu / \rho$ is the kinematic viscosity, $\omega$ is the frequency of the sphere oscillations, and $a$ is the sphere radius. This is readily satisfied for sufficiently high kinematic viscosity and/or sufficiently low frequency; hence the constraints are not mutually exclusive when interpreted physically.

With the kinematic problem, where the relative swimmer motions are unchanged in the different media, we find that viscoelasticity has no influence; however, the mobility problem, whereby the actuation forces are prescribed, clearly indicates substantial effects. In particular, we have compared swimming behavior for a given, sinusoidal, and nonreciprocal, forcing of the three-sphere swimmer within a Newtonian fluid and within a viscoelastic medium, the latter with standard parameter constraints associated with a polymeric fluid. This leads to the prediction that within the polymeric viscoelastic medium, the swimmer is both more efficient and moves further in one beat cycle at leading nontrivial orders of the asymptotic expansion.

While improvements in swimming and efficiency are observed by Teran et al. [11] for the kinematic problem associated with undulating sheet swimmers at Deborah numbers of order one, the influence of viscoelasticity is very different. In particular, Teran $e t$ al. reported a peak of optimal efficiency and swimming speed with a Deborah number of unity due to stresses induced by the fluid aft to the swimmer, suggesting a resonance between the undulation frequency and the fluid at optimality. In contrast, no swimmer-medium resonance is observed here even though the parameter regime contains order one Deborah numbers. While Teran et al.'s [11] study is for large amplitude displacements, resonance phenomena are regularly observed for small amplitude approximations with prescribed forcings, as with the simple harmonic oscillator. In turn, this suggests that the absence of resonance in this study is not solely a consequence of the small amplitude oscillations used to facilitate an asymptotic investigation. Furthermore, Fu et al. [13] for example report that nonlinear viscoelastic effects reduce swimmer speeds for an infinite undulating cylinder. Consequently, the current study reinforces the observations of the current literature that the responses of inertialess swimmers to non-Newtonian rheologies do not appear to be systematic, but vary on a case-by-case basis. In particular, this emphasizes that caution must be used when extrapolating physical insight from simplified models of microswimmers in nontrivial rheologies to other systems, such as swimming cells.

In addition, our predictions are analogous to the fully linear study of Fulford and Katz [9] and would be predicted on consideration of simply linear viscoelastic constitutive relations: here, nonlinear viscoelasticity does not alter the force balance even though its dynamically nontrivial. Thus, firstly, while it is commonly observed from studies of the kinematic problem that linear viscoelasticity is insufficient to capture even leading-order effects in the study of microswimming [26], this is clearly not reflected here. This is despite the observation that the scalings in this paper indicate nonlinear viscoelasticity is important, noting that higher orders in the perturbative expansion must be considered to capture the net motion of the swimmer. Thus, the fact linear viscoelasticity is sufficient to deduce the leading-order swimming behavior in this study only emerges a posteriori; the extent to which this may generalize is unclear and merits further study if only to understand when simple, fully linear, methods might be exploited in some settings for understanding inertialess swimming in rheological media.

\section{ACKNOWLEDGMENTS}

This publication is based on work supported by Award No. KUK-C1-013-04, made by King Abdullah University of Science and Technology (KAUST). We are grateful to Qixuan Wang (School of Mathematics, University of Minnesota) for discussions in the early phases of this work.

\section{APPENDIX}

Here we investigate a flow field, $\mathbf{v}_{1}^{(\alpha)}$, outside a unit sphere satisfying

$$
\begin{aligned}
-\nabla s_{1}^{(\alpha)}+\nabla^{2} \mathbf{v}_{1}^{(\alpha)} & =\nabla \cdot \eta^{(\alpha)}, \quad \nabla \cdot \mathbf{v}_{1}^{(\alpha)}=0, \\
\mathbf{v}_{1}^{(\alpha)} & =\mathbf{0} \quad \text { on } \quad|\mathbf{x}|=1,
\end{aligned}
$$

for some pressure function $s_{1}^{(\alpha)}$, where

$$
\begin{aligned}
\eta^{(\alpha)}:= & \left(1+\Gamma \frac{\partial}{\partial t}\right)^{-1}\left[\mathbf{u}_{0}^{(\alpha)} \cdot \nabla\left[\Lambda \boldsymbol{\tau}_{0}^{(\alpha)}-\Gamma \mathcal{E}_{0}^{(\alpha)}\right]\right. \\
& -\left\{\left(\nabla \mathbf{u}_{0}^{(\alpha)}\right)^{T} \cdot\left[\Lambda \boldsymbol{\tau}_{0}^{(\alpha)}-\Gamma \mathcal{E}_{0}^{(\alpha)}\right]\right. \\
& \left.+\left[\Lambda \boldsymbol{\tau}_{0}^{(\alpha)}-\Gamma \mathcal{E}_{0}^{(\alpha)}\right] \cdot \nabla \mathbf{u}_{0}^{(\alpha)}\right\} \\
& \left.-\mathbf{U}_{0}^{(\alpha)} \cdot \nabla\left[\Lambda \boldsymbol{\tau}_{0}^{(\alpha)}-\Gamma \mathcal{E}_{0}^{(\alpha)}\right]\right]
\end{aligned}
$$

which is a known function as $\boldsymbol{\tau}_{0}^{(\alpha)}$ and $\mathcal{E}_{0}^{(\alpha)}$ in turn are defined via the leading-order flow field $\mathbf{u}_{0}^{(\alpha)}$ from Eqs. (55), as given by Eqs. (57) and (59).

In particular, we aim to show that $\mathbf{v}_{1}^{(\alpha)}$ satisfies the following conditions on the boundary of the unit sphere and the boundaries of two other unit spheres located at $O(1 / \delta)$ distances away,

$$
\begin{array}{lll}
\mathbf{v}_{1}^{(\alpha)}=\mathbf{0} \quad \text { on } & |\mathbf{x}|=1, \quad \beta=\alpha, \\
\mathbf{v}_{1}^{(\alpha)} \sim \delta^{2} \mathbf{A}^{(\alpha)} & \text { on } \quad\left|\mathbf{x}-\mathbf{x}^{(\beta)}\right|=1, \quad \beta \neq \alpha .
\end{array}
$$

When solving the $\mathbf{v}_{1}^{(\alpha)}$ equations, we consider only the leadingorder terms of $\boldsymbol{\nabla} \cdot \boldsymbol{\eta}^{(\alpha)}$ in Eq. (A1) as this will be shown to be sufficient in proving (A3). To obtain this, we note that the leading order expression for $\mathbf{u}_{0}^{(\alpha)}(\mathbf{x})$ from Eqs. (57) and (59) is

$$
\mathbf{u}_{0}^{(\alpha)}(\mathbf{x}) \sim-\mathbf{U}_{0}^{(\alpha)}+\mathbf{G}(\mathbf{x}, \mathbf{0}) \mathbf{U}_{0}^{(\alpha)}+O(\delta),
$$


where, as before, $\mathbf{G}$ is the singularity combination of a Stokeslet, $\mathbf{S}$, and potential dipole, $\mathbf{D}$, of the form

$$
\mathbf{G}\left(\mathbf{x}, \mathbf{x}^{(\alpha)}\right):=\frac{3}{4} \mathbf{S}\left(\mathbf{x}, \mathbf{x}^{(\alpha)}\right)-\frac{1}{4} \mathbf{D}\left(\mathbf{x}, \mathbf{x}^{(\alpha)}\right) .
$$

This gives the leading-order expression for $\boldsymbol{\eta}^{(\alpha)}$ as

$$
\begin{aligned}
\boldsymbol{\eta}^{(\alpha)} \approx & \left(1+\Gamma \frac{\partial}{\partial t}\right)^{-1}\left[\mathbf{G}(\mathbf{x}, \mathbf{0}) \mathbf{U}_{0}^{(\alpha)} \cdot \nabla\left[\Lambda \boldsymbol{\tau}_{0}^{(\alpha)}-\Gamma \mathcal{E}_{0}^{(\alpha)}\right]\right. \\
& -\left\{\left(\nabla \mathbf{G}(\mathbf{x}, \mathbf{0}) \mathbf{U}_{0}^{(\alpha)}\right)^{T} \cdot\left[\Lambda \boldsymbol{\tau}_{0}^{(\alpha)}-\Gamma \mathcal{E}_{0}^{(\alpha)}\right]\right. \\
& \left.+\left[\Lambda \boldsymbol{\tau}_{0}^{(\alpha)}-\Gamma \mathcal{E}_{0}^{(\alpha)}\right] \cdot \nabla \mathbf{G}(\mathbf{x}, \mathbf{0}) \mathbf{U}_{0}^{(\alpha)}\right\} \\
& \left.-2 \mathbf{U}_{0}^{(\alpha)} \cdot \nabla\left[\Lambda \boldsymbol{\tau}_{0}^{(\alpha)}-\Gamma \mathcal{E}_{0}^{(\alpha)}\right]\right],
\end{aligned}
$$

where, from Eqs. (55) and (A4),

$$
\begin{aligned}
\mathcal{L}\left[\boldsymbol{\tau}_{0}^{(\alpha)}\right] & =\mathcal{E}_{0}^{(\alpha)}, \\
& \approx\left(\nabla \mathbf{G}(\mathbf{x}, \mathbf{0}) \mathbf{U}_{0}^{(\alpha)}\right)^{T}+\nabla \mathbf{G}(\mathbf{x}, \mathbf{0}) \mathbf{U}_{0}^{(\alpha)},
\end{aligned}
$$

recalling (56),

$$
\mathcal{L}:=\left(1+\Gamma \frac{\partial}{\partial t}\right)^{-1}\left(1+\Lambda \frac{\partial}{\partial t}\right)
$$

As detailed in the main text, we seek a solution where initial transient behavior has decayed and is thus perfectly periodic, which entails the inverse operator in the definition of $\mathcal{L}$ is uniquely defined.

The divergence of $\boldsymbol{\eta}^{(\alpha)}$, in tensor notation (index summation assumed) where $r=|\mathbf{x}|, r_{i}=x_{i}$ and $\mathbf{U}_{0}^{(\alpha)}$ has components $U_{i}^{(\alpha)}$, can be written in the form

$$
\begin{aligned}
{\frac{\partial \eta_{i j}}{\partial r_{j}}}^{(\alpha)}= & C_{i j k}\left(1+\Gamma \frac{\partial}{\partial t}\right)^{-1}\left[U_{j}^{(\alpha)}\left(\Lambda \mathcal{L}^{-1}-\Gamma\right)\left[U^{(\alpha)}\right]_{k}\right] \\
& -\frac{\partial}{\partial r_{i}}\left(1+\Gamma \frac{\partial}{\partial t}\right)^{-1}\left(2 U_{k} \frac{\partial\left(\Lambda \mathcal{L}^{-1}-\Gamma\right) p_{0}{ }^{(\alpha)}}{\partial r_{k}}\right)
\end{aligned}
$$

We take the $r_{3}$ direction to be along the axis of the swimmer, $\mathbf{e}_{z}$, so that $r_{3}=z$ and $U_{i}^{(\alpha)}=U^{(\alpha)} \delta_{i 3}$. Noting that $\mathbf{G}$ is solely a function of radial components, we redimensionalize to a sphere of radius $a$ in order to inspect powers of $a / r$ in the tensor $\mathbf{G}$, and hence identify the form of equation (A6). Upon subsequent nondimensionalization, we can deduce that the tensor $C_{i j k}$ in equation (A9) for the divergence of $\boldsymbol{\eta}^{(\alpha)}$ takes the form

$$
\begin{gathered}
C_{i j k}\left(1+\Gamma \frac{\partial}{\partial t}\right)^{-1}\left[U_{j}^{(\alpha)}\left(\Lambda \mathcal{L}^{-1}-\Gamma\right)\left[U^{(\alpha)}\right]_{k}\right] \\
=\bar{C}_{i}\left(1+\Gamma \frac{\partial}{\partial t}\right)^{-1}\left\{U^{(\alpha)}\left(\Lambda \mathcal{L}^{-1}-\Gamma\right)\left[U^{(\alpha)}\right]\right\},
\end{gathered}
$$

where $\bar{C}_{i}$ is given in terms of obtainable constants $A_{m n}$ (whose values are not required for this calculation),

$$
\begin{aligned}
\bar{C}_{i}= & \left(\frac{A_{11}}{r^{2}}+\frac{A_{12}}{r^{4}}+\frac{A_{13}}{r^{6}}\right) \frac{r_{i} z^{2}}{r^{6}} \\
& +\left(\frac{A_{21}}{r^{2}}+\frac{A_{22}}{r^{4}}+\frac{A_{23}}{r^{6}}\right) \frac{r_{i}}{r^{4}} \\
& +\left(\frac{A_{31}}{r^{2}}+\frac{A_{32}}{r^{4}}+\frac{A_{33}}{r^{6}}\right) \frac{\delta_{i 3} z}{r^{4}} .
\end{aligned}
$$

One can show that the $A_{1 j}$ terms in Eq. (A11) of the form $r_{i} z^{2} / r^{k}$ can be expressed as a $\delta_{i 3} z / r^{k}$ term, the same form as the $A_{3 j}$ terms, plus a gradient of a radial function. The $A_{2 j}$ terms in Eq. (A11) of the form $r_{i} / r^{k}$ can also be expressed as a gradient of radial function, so that $\bar{C}_{i}$ can be written

$$
\begin{aligned}
\bar{C}_{i}= & {\left[\frac{\left(A_{31}+A_{11} / 3\right)}{r^{2}}+\frac{\left(A_{32}+A_{12} / 4\right)}{r^{4}}+\frac{\left(A_{33}+A_{13} / 5\right)}{r^{6}}\right] } \\
& \times \frac{\delta_{i 3} z}{r^{4}}+\frac{\partial \psi}{\partial r_{i}},
\end{aligned}
$$

where $\psi$ includes all the radial functions that can be expressed as a gradient; note that $\psi$ is not required explicitly. Substituting this into Eq. (A9) gives the full expression for the divergence of $\eta^{(\alpha)}$,

$$
{\frac{\partial \eta_{i j}}{\partial r_{j}}}^{(\alpha)}=\left[\sum_{m=1}^{3} \frac{\zeta_{m}^{\alpha}}{r^{2 m}}\right] \frac{\delta_{i 3} z}{r^{4}}+\frac{\partial \bar{\psi}}{\partial r_{i}}
$$

where $\bar{\psi}=\psi-2 U_{k}{\frac{\partial p_{0}}{\partial r_{k}}}^{(\alpha)}$ and

$$
\begin{aligned}
\zeta_{m}^{\alpha}= & \left(A_{3 m}+\frac{A_{1 m}}{2+m}\right)\left(1+\Gamma \frac{\partial}{\partial t}\right)^{-1} \\
& \times\left\{U^{(\alpha)}\left(\Lambda \mathcal{L}^{-1}-\Gamma\right)\left[U^{(\alpha)}\right]\right\} .
\end{aligned}
$$

The $\mathbf{v}_{1}^{(\alpha)}$ problem (A1) is thus

$$
\begin{aligned}
-\nabla \bar{s}_{1}^{(\alpha)}+\nabla^{2} \mathbf{v}_{1}^{(\alpha)} & =\left[\sum_{m=1}^{3} \frac{\zeta_{m}^{\alpha}}{r^{2 m}}\right] \frac{z \mathbf{e}_{z}}{r^{4}}, \\
\nabla \cdot \mathbf{v}_{1}^{(\alpha)} & =0, \quad \mathbf{v}_{1}^{(\alpha)}=\mathbf{0} \quad \text { on } \quad r=1,
\end{aligned}
$$

where $\bar{s}_{1}^{(\alpha)}=s_{1}^{(\alpha)}+\bar{\psi}$ is the modified pressure term, absorbing any gradients of radial functions.

The particular integral, denoted by superscript $p$, satisfies

$$
-\nabla s^{p}+\nabla^{2} \mathbf{v}^{p}=\left[\sum_{m=1}^{3} \frac{\zeta_{m}^{\alpha}}{r^{2 m}}\right] \frac{z \mathbf{e}_{z}}{r^{4}}, \quad \nabla \cdot \mathbf{v}^{p}=0,
$$

while the complementary function, superscript $c$, satisfies

$$
\begin{aligned}
-\nabla s^{c}+\nabla^{2} \mathbf{v}^{c} & =\mathbf{0}, \quad \nabla \cdot \mathbf{v}^{c}=0, \\
\mathbf{v}^{c} & =-\mathbf{v}^{p} \quad \text { on } \quad r=1,
\end{aligned}
$$

where $\mathbf{v}_{1}^{(\alpha)}=\mathbf{v}^{c}+\mathbf{v}^{p}$ and $\bar{s}_{1}^{(\alpha)}=s^{p}+s^{c}$. From the particular integral equations (A15), one can deduce a form $s^{p}$ and hence $\mathbf{v}^{p}$, giving, after some lengthy algebra,

$$
\begin{aligned}
\mathbf{v}^{p}= & \sum_{m=1}^{3} \frac{\zeta_{m}^{\alpha}}{(2 m+2)}\left\{\frac{-z \mathbf{e}_{z}}{(2 m-1) r^{2 m+2}}-\frac{1}{(2 m-1)(2 m-3)}\right. \\
& \left.\times\left[\frac{2 z \mathbf{e}_{z}}{r^{2 m+2}}+\frac{\mathbf{e}_{r}}{r^{2 m+1}}-\frac{(2 m+2) z^{2} \mathbf{e}_{r}}{r^{2 m+3}}\right]\right\},
\end{aligned}
$$

which, on $r=1, z=\cos \theta$ in standard spherical polar coordinates, becomes

$$
\left.\mathbf{v}^{p}\right|_{r=1}=\sum_{m=1}^{3} \frac{\zeta_{m}^{\alpha}\left[\left(3 \cos ^{2} \theta-1\right) \mathbf{e}_{r}+(2 m-1) \sin \theta \cos \theta \mathbf{e}_{\theta}\right]}{(2 m+2)(2 m-1)(2 m-3)},
$$


This gives us our boundary condition for the complementary problem (A16), motivating the form of $\mathbf{v}^{c}$, in particular being a linear combination of Stokes' flow solutions,

$$
\begin{aligned}
& \mathbf{v}_{1}^{c}=\frac{\cos ^{2} \theta \mathbf{e}_{r}}{r^{2}}, \quad \mathbf{v}_{2}^{c}=\frac{\mathbf{e}_{r}}{r^{2}}, \\
& \mathbf{v}_{3}^{c}=\frac{\left(3 \cos ^{2} \theta-1\right) \mathbf{e}_{r}}{r^{4}}+\frac{2 \cos \theta \sin \theta \mathbf{e}_{\theta}}{3 r^{4}},
\end{aligned}
$$

The dominant contribution to $\mathbf{v}_{1}^{(\alpha)}$ as $r$ increases away from sphere $\alpha$ arises from the complementary function, and is $O\left(1 / r^{2}\right)$. In particular, on another sphere an $O(1 / \delta)$ distance away,

$$
\mathbf{v}_{1}^{(\alpha)} \sim \delta^{2} \mathbf{A}^{(\beta)} \text { on }\left|\mathbf{x}-\mathbf{x}^{(\beta)}\right|=1, \quad \beta \in\{1,2,3\},
$$

for constant vectors $\mathbf{A}^{(\beta)}$, where $\mathbf{A}^{(\alpha)}=\mathbf{0}$, noting that $\mathbf{e}_{r} \sim \mathbf{e}_{z}$ and $\cos \theta \sim 1$ at leading order in $\delta$.
[1] D. J. Smith, E. A. Gaffney, and J. R. Blake, Proc. R. Soc. A 465 2417 (2009).

[2] N. Liron and S. Mochon, J. Fluid Mech. 75, 593 (1976).

[3] E. A. Gaffney, H. Gadêlha, D. J. Smith, J. R. Blake, and J. C. Kirkman-Brown, Annu. Rev. Fluid Mech. 43, 501 (2011).

[4] P. Y. Tam, D. F. Katz, and S. A. Berger, Biorheology 17, 465 (1980).

[5] A. Ivic, H. Onyeaka, A. Girling, I. Brewis, B. Ola, N. Hammadieh, S. Papaioannou, and C. Barratt, Hum. Reprod 17, 143 (2002).

[6] A. Najafi and R. Golestanian, Phys. Rev. E 69, 062901 (2004).

[7] M. Leoni, J. Kotar, B. Bassetti, P. Cicuta, and M. C. Lagomarsino, Soft Matter 5, 472 (2009).

[8] R. Zargar, A. Najafi, and M. Miri, Phys. Rev. E. 80, 026308 (2009).

[9] G. R. Fulford, D. F. Katz, and R. L. Powell, Biorheology 35, 296 (1998).

[10] R. E. Johnson, J. Fluid Mech. 99, 411 (1980).

[11] J. Teran, L. Fauci, and M. Shelley, Phys. Rev. Lett. 104, 38101 (2010).

[12] H. C. Fu, T. R. Powers, and C. W. Wolgemuth, Phys. Rev. Lett. 99, 258101 (2007).

[13] H. C. Fu, C. W. Wolgemuth, and T. R. Powers, Phys. Fluids 21, 033102 (2009).

[14] E. M. Purcell, Am. J. Phys. 45, 3 (1977).

[15] E. Lauga, Europhys. Lett. 86, 64001 (2009).

[16] R. Golestanian and A. Ajdari, Phys. Rev. E 77, 036308 (2008).
[17] C. M. Pooley, G. P. Alexander, and J. M. Yeomans, Phys. Rev. Lett. 99, 228 (2007).

[18] G. P. Alexander, C. M. Pooley, and J. M. Yeomans, J. Phys.: Condens. Matter 21, 204108 (2009).

[19] T. D. Montenegro-Johnson, A. A. Smith, D. J. Smith, D. Loghin, and J. R. Blake, Eur. Phys. J. E: Soft Matter and Biological Physics 35, 1 (2012).

[20] G. Batchelor, An Introduction to Fluid Dynamics (Cambridge University Press, Cambridge, 1967).

[21] J. G. Oldroyd, Proc. R. Soc. London A 200, 523 (1950).

[22] D. F. James, Annu. Rev. Fluid Mech. 41, 129 (2009).

[23] R. B. Bird, R. C. Armstrong, and O. Hassager, Fluid Mechanics, Vol. 1 of Dynamics of Polymeric Liquids (Wiley, New York, 1977).

[24] G. J. Elfring, O. S. Pak, and E. Lauga, J. Fluid Mech. 646, 505 (2010).

[25] R. B. Bird and J. M. Wiest, Annu. Rev. Fluid Mech. 27, 169 (1995).

[26] E. Lauga, Phys. Fluids 19, 083104 (2007).

[27] D. J. Earl, C. M. Pooley, J. F. Ryder, I. Bredberg, and J. M. Yeomans, J. Chem. Phys. 126, 064703 (2007).

[28] S. Kim and S. J. Karrila, Microhydrodynamics (ButterworthHeinemann, New York, 1991).

[29] C. Pozrikidis, Boundary integral and singularity methods for linearized viscous flow (Cambridge University Press, Cambridge, 1992).

[30] M. J. Lighthill, Commun. Pure Appl. Math. 5, 109 (1952). 